\title{
Stability and transition in pitching wings
}

by

\author{
Prabal S. Negi
}

November 2019

Technical Reports

Royal Institute of Technology

Department of Mechanics

SE-100 44 Stockholm, Sweden 
Akademisk avhandling som med tillstånd av Kungliga Tekniska högskolan i Stockholm framlägges till offentlig granskning för avläggande av teknologie doktorsexamen fredagen den 22 November 2019 kl 10:00 i sal F3, Kungliga Tekniska högskolan, Lindstedtsvägen 26, Stockholm.

TRITA-SCI-FOU 2019:46

ISBN 978-91-7873-348-4

Cover: Space-time plot of the suction-side wall-shear stress for a pitching laminar airfoil at $\operatorname{Re}_{c}=7.5 \times 10^{5}$. The vertical axis represents chord-wise location and the horizontal axis represents time. Blue colors represent low or negative shear-stress and brown colors represent high shear-stress. Black contours represent zero shear-stress values.

(C)Prabal S. Negi 2019

Universitetsservice US-AB, Stockholm 2019 
आँखों में सपने लिये

घर से हम चल तो दिये

जाने ये राहें अब ले जाएँगी कहाँ

मिट्टि की खुशबू आए

पत्रकों पे आंसू लाए

पलकों पे रह जायेगा यादों का जहाँ मंज़िल नयी है अंजाना है कारवां चलना अकेले है यहाँ

- शान
तन्हां दिल





\title{
Stability and transition in pitching wings
}

\section{Prabal S. Negi}

Department of Mechanics, Linné FLOW Centre and Swedish e-Science Research Centre (SeRC), KTH Royal Institute of Technology,

SE-100 44 Stockholm, Sweden

\begin{abstract}
The aeroelastic stability of airplanes is one of the most important aspects of airplane design. Flutter or divergence instabilities arising out of the interaction of fluid forces and structural elasticity must be avoided by design or through the limitation of the flight envelope. Classical unsteady theories have been established since the 1930s however, recent investigations with laminar wings and in transitional flows have found the theories to be unreliable in these regimes. The current work investigates the flow around unsteady airfoils in these flow regimes.

A linear framework for the stability analysis of fluid-structure-interaction (FSI) problems is derived and validated. The derived formulation is then used to investigate the changes in the structural sensitivity of an eigenvalue for an oscillating cylinder, which is found to change significantly when the fluid and structural systems are close to resonance.

The linear stability analysis is then applied to investigate the aeroelastic stability of a NACA0012 airfoil with a free pitch-deegree-of-freedom at transitional Reynolds numbers. The stability results of the coupled FSI system are found to be in good agreement with previously performed experimental results and were able to predict the onset of aeroelastic pitch-oscillations.

The boundary layer evolution for a natural laminar flow airfoil undergoing forced small-amplitude pitch-oscillations is investigated at $R_{c}=7.5 \times 10^{5}$. Large changes in laminar-to-turbulent transition location are found throughout the pitch cycle which cause a non-linear aerodynamic force response. The origins of the non-linear unsteady aerodynamic response is explained on the basis of the phase-lagged quasi-steady evolution of the boundary layer. A simple empirical model is developed using the phase-lag concept to model the unsteady aerodynamic forces which fits the experimental data very well.

On the other hand, the forced pitching investigation at $R e_{c}=1.0 \times 10^{5}$ for the same airfoil found abrupt changes in transition during the pitch-cycle. A local stability analysis in the reverse flow region indicates that the stability characteristics of the LSB change character from convective to absolute, and it is conjectured that this change in stability characteristics may be the cause of abrupt changes in boundary-layer transition.
\end{abstract}

Keywords: boundary layers, unsteady aerodynamics, aeroelasticity, stability, fluidstructure-interaction 


\section{Stabilitet och omslag av flödet över oscillerande vingar}

\section{Prabal S. Negi}

Institutionen för Mekanik, Linné FLOW Centre och Swedish e-Science Research Centre (SeRC), Kungliga Tekniska högskolan

SE-100 44 Stockholm, Sverige

\section{Sammanfattning}

Aeroelastisk stabilitet är en av de viktigaste aspekterna vid flygplans utformning. Vibrations- eller divergensinstabiliteter, som uppstår till följd av växelverkan mellan fluidkrafter och strukturell elasticitet, måste undvikas genom modifiering av konstruktionen eller genom begränsningar i flygbanan. Teorier för icke-stationär aerodynamik har funnits sedan 1930-talet, men nyligen genomförda undersökningar med laminära vingar under förhållanden där strömningen genomgår laminärt-turbulent omslag, har visat att dessa teorier inte är pålitliga i dessa regimer. I detta arbete undersöks strömningen kring icke-stationära vingar under sådana flödesförhållanden.

Ett linjärt ramverk för stabilitetsanalys av fluid-struktur interaktion (FSI) härleds och valideras. Den härledda formuleringen används sedan för att undersöka förändringen i känslighet hos ett egenvärde för en oscillerande cylinder, vilken visar sig vara avsevärd när fluiden och strukturen är nära resonans.

Den linjära stabilitetsanalysen tillämpas sedan för att undersöka den aeroelastiska stabiliteten hos en NACA0012-vingprofil med en rotations-frihetsgrad vid transitionella Reynoldstal. Stabilitetsresultaten för det kopplade FSI-systemet visar sig vara i god överensstämmelse med resultat från tidigare utförda experiment och kapabla att förutsäga begynnelsen av aeroelastiska rotationssvängningar.

Utvecklingen av gränsskiktet över en laminär vingprofil som genomgår en påtvingad rotation med små amplituder undersöks vid $R e_{c}=7.5 \times 10^{5}$. Stora förändringar hittas i positionen för omslagslaget mellan laminär och turbulent strömning under en rotationscykel, vilket orsakar en icke-linjär aerodynamisk respons. Ursprunget till den icke-linjära och icke-stationära aerodynamiska responsen förklaras genom en fasfördröjd kvasistationär utveckling av gränsskiktet. En enkel empirisk modell utvecklas med hjälp av fasfördröjningskonceptet för att modellera de icke-stationära aerodynamiska krafterna, vilka överensstämmer väl med de experimentella mätresultaten.

$\AA$ andra sidan visade analysen av samma vingprofil vid $R e_{c}=1.0 \times 10^{5}$ abrupta förändringar för omslagslaget mellan laminärt och turbulent flöde under en rotationscykel då den utsattes för påtvingade svängningar. En lokal stabilitetsanalys i området med motriktad strömning indikerar att stabilitetsegenskaperna hos den laminära separationsbubblan ändrar karaktär från konvektiv till absolut. Slutsatsen dras att denna förändring i stabilitetsegenskaper kan vara orsaken till de abrupta förändringarna i omslaget mellan laminär och turbulent strömning i gränsskiktet.

Nyckelord: gränsskikt, ickestationär aerodynamik, aeroelasticitet, stabilitet, fluidstruktur interaktion 


\section{Preface}

This thesis deals with the numerical investigation of stability and boundary-layer transition in an unsteady wing undergoing pitch oscillations. The investigation of the unsteady phenomenon is confined to two particular flow scenarios. The first is the lowto-moderate Reynolds numbers in which the flow over the wing is in the transitional regime. This comprises of investigated chord-based Reynolds numbers lying in the range of $40,000 \leq R e_{c} \leq 100,000$. The second is an investigation at a much higher Reynolds number of $R e_{c}=750,000$, for a natural laminar flow airfoil. While the two flow regimes are vastly different a common thread runs through them. In both the flow regimes the unsteady flow dynamics are in large part dictated by the varying transition and separation characteristics of the unsteady boundary layer. The result of the dominating role played by the unsteady boundary-layer is that standard linear unsteady aerodynamic theories fail to predict some of the phenomenon observed in experimental investigations of unsteady airfoils in these regimes. While aeroelastic investigations have a long history with some of the theoretical work dating back to the 1920s, systematic investigations on transitional Reynolds numbers and laminar airfoils has only emerged within the last decade with the seminal works of Poirel et al. (2008) on transitional Reynolds numbers and Mai \& Hebler (2011) on laminar airfoils. The work in this thesis is largely an attempt to explain some of the observations emerging out of the aforementioned studies.

A brief introduction of the basic concepts, methods and overview of the important results is presented in the first part. The second part contains six articles referred to as papers. The first is a technical report on the stability of the numerical method in use. Paper two to five deal with the heart of the matter which is on "stability and transition in pitching wings". The final paper deals with the development of a turbulent boundary layer over a stationary wing section. The fourth paper is an extended version of the conference paper presented at the AIAA Aviation forum, Applied aerodynamics conference, June 2018, Atlanta, Georgia, USA. All articles have been adjusted to comply with the present thesis format for consistency, but the contents have not been altered as compared with the original counterpart.

Paper 1. Negi, Schlatter \& Henningson. A re-examination of filter-based stabilization for spectral-element methods. Tech. Rep.

Paper 2. Negi, Hanifi \& Henningson. Global stability of rigid-body-motion fluidstructure-interaction problems. Manuscript.

Paper 3. Negi, Hanifi \& Henningson. On the onset of aeroelastic pitch-oscillations of a NACA0012 wing at transitional Reynolds numbers. Manuscript.

Paper 4. Negi, Hanifi \& Henningson. Linear and non-linear response of a laminar airfoil subject to small amplitude pitch-oscillations. Manuscript. 
Paper 5. Negi, Vinuesa, Schlatter, Hanifi \& Henningson, 2018. Unsteady aerodynamic effects in small-amplitude pitch oscillations of an airfoil. Int. J. Heat \& Fluid Flow 71, 378-391.

Paper 6. Vinuesa, Negi, Atzori, Hanifi, Henningson \& Schlatter, 2018. Turbulent boundary layers around wing sections up to $R e_{c}=1,000,000$. Int. J. Heat \& Fluid Flow 72, 86-99.

November 2019, Stockholm

Prabal S. Negi 


\section{Division of work between authors}

The main advisor for the project is Professor Dan S. Henningson (DH). Docent Ardeshir Hanifi (AH) and Professor Philipp Schlatter (PS) act as co-advisors.

Paper 1. The matlab code has been developed by Prabal S. Negi (PSN). The numerical simulations have been set-up and run by PSN. The paper has been written by PSN with feedback from PS and DH.

Paper 2. The mathematical formulation is developed by PSN with feedback from AH and DH. The numerical validations have been performed by PSN. The paper has been written by PSN with feedback from $\mathrm{AH}$ and $\mathrm{DH}$.

Paper 3. The numerical simulation has been set-up and run by PSN. The paper has been written by PSN with feedback from $\mathrm{AH}$ and $\mathrm{DH}$.

Paper 4. The numerical simulation has been set-up and run by PSN. The empirical model has been developed by PSN. The paper has been written by PSN with feedback from $\mathrm{AH}$ and $\mathrm{DH}$.

Paper 5. The numerical simulations for channel flows and pitching airfoil have been set-up and run by PSN. Numerical simulations for LES validation on wing section has been setup by PSN and run by Ricardo Vinuesa (RV). The paper has been written by PSN with feedback from RV, AH, PS and DH.

Paper 6. The numerical simulation for the $R e_{c}=1,000,000$ case has been set-up by PSN and run by RV. The domain validation was performed by PSN. Marco Atzori (MA) performed the simulations for $R e_{c}=200,000$. The paper has been written by RV with feedback from MA, PSN, AH, DH and PS.

\section{Conferences}

Part of the work in this thesis has been presented at the following international conferences. The presenting author is underlined.

P. S. Negi, A. HANIFI, \& D. S. Henningson. Global stability of fluid-structure-interaction problems. IUTAM Symposium on laminar-turbulent transition. London, United Kingdom, 2019.

P. S. Negi, A. Hanifi, \& D. S. Henningson. High-fidelity simulations of the unsteady response of an NLF airfoil. Aerospace Technology Congress. Stockholm, Sweden, 2019.

D. S. Henningson, P. S. Negi, S. R. Vinuesa, M. Hosseini, A. Hanifi \& P. Schlatter. Large scale numerical experiments of pitching wings and the role of laminar-turbulent transition. Svenska Mekanikdagar. Stockholm, Sweden, 2019.

P. S. Negi, A. Hanifi, \& D. S. Henningson. LES of the unsteady response of natural laminar flow airfoil. Hi-Fidelity Industrial LES/DNS Symposium (HiFiLED). Brussels, Belgium, 2018. 
P. S. Negi, A. Hanifi, \& D. S. Henningson. LES of the unsteady response of natural laminar flow airfoil. AIAA Aviation Forum, Applied Aerodynamics Conference. Atlanta, Georgia, USA, 2018.

P. S. Negi, R. Vinuesa, M. Atzori, P. Schlatter, A. Hanifi, \& D. S. Henningson. Unsteady aerodynamic simulations. The ninth annual meeting of SeRC. Upplands-Bro N, Sweden, 2018.

P. S. Negi, R. Vinuesa, A. Hanifi, P. Schlatter \& D. S. Henningson. Large-eddy simulations of a wing section undergoing small-amplitude pitch oscillations. $16^{\text {th }}$ European Turbulence Conference (ETC). Stockholm, Sweden, 2017.

P. S. Negi, R. Vinuesa, A. Hanifi, P. Schlatter \& D. S. Henningson. Unsteady aerodynamic effects in small-amplitude pitch oscillations of an airfoil. $10^{\text {th }}$ Int. Symp. on Turbulence \& Shear Flow Phenomenon (TSFP-10). Chicago, USA, 2017.

R. Vinuesa, P. S. Negi, A. Hanifi, D. S. Henningson \& P. Schlatter. High-fidelity simulations of the flow around wings at high Reynolds numbers. $10^{\text {th }}$ Int. Symp. on Turbulence \& Shear Flow Phenomenon (TSFP-10). Chicago, USA, 2017.

P. S. Negi, R. Vinuesa, P. Schlatter, A. Hanifi, \& D. S. Henningson. Relaxation-term filertering for SEM. $5^{\text {th }}$ Nek user's meeting. Cambridge, USA, 2016. 


\title{
Contents
}

\begin{abstract}
$\quad$ v
Sammanfattning vi vi

Preface vii
\end{abstract}

Part I - Overview and summary

Chapter 1. Introduction 1

1.1. A short history 1

1.2. Aeroelasticity and aircraft design 2

1.3. Boundary layers over a stationary wing 6

Chapter 2. Numerical Method 8

2.1. Numerical Discretization 8

2.2. Relaxation-term large-eddy simulation (RT-LES) 9

2.3. Arbitrary-Lagrangian-Eulerian (ALE) 10

2.4. Fluid-Structure coupling $\quad 12$

2.5. Free-stream turbulence 13

$\begin{array}{lll}\text { Chapter 3. Overview of results } & 15\end{array}$

3.1. Introduction 15

3.2. Linear stability of fluid-structure-interaction problems 15

3.3. Onset of pitch-oscillations at transitional Reynolds numbers 20

3.4. Forced response of pitching wings 21

Chapter 4. Conclusions and outlook 30

4.1. Conclusions 30

4.2. Outlook 31

Acknowledgements 33

$\begin{array}{ll}\text { Bibliography } & 35\end{array}$ 
Part II - Papers

Summary of the papers

Paper 1. A re-examination of filter-based stabilization for spectralelement methods

Paper 2. Global stability of rigid-body-motion fluid-structureinteraction problems

Paper 3. On the onset of aeroelastic pitch-oscillations of a NACA0012 wing at transitional Reynolds numbers 107

Paper 4. Linear and non-linear response of a laminar airfoil subject to small amplitude pitch-oscillations

Paper 5. Unsteady aerodynamic effects in small-amplitude pitch oscillations of an airfoil

Paper 6. Turbulent boundary layers around wing sections up to $R e_{c}=1,000,000$ 


\section{Part I}

\section{Overview and summary}



CHAPTER 1

\section{Introduction}

\subsection{A short history}

The first sustained flight by the Wright brothers in 1903 marked a historic day in human achievement and ingenuity. Momentous as the achievement was, the Wright brothers did not truly invent the modern airplane. Their achievements were the fruition of nearly a century of aeronautical research, starting perhaps with Sir George Cayley, who is considered the "father of aerial navigation" (Gibbs-Smith 1962). The principal components of the modern aircraft were laid down by George Cayley as early as 1799. Prior to Cayley, the ideas for mechanical flight tended towards flapping wings, where the flapping motion produced both propulsion and lift. George Cayley was the first to break the unsuccessful chain of thought and separated the two aspects of flight into distinct systems. His first three papers "On Aerial Navigation" published in Nicholason's Journal of Natural Philosophy, Chemistry and the Arts on November 1809, February and March 1810 (Cayley 1809-10) mark some of the most important works in aeronautical history. In the works, Cayley states the principle of lift generation i.e. the formation of a low pressure region on the upper surface of the wing. His paper elaborates on the separation of lift from propulsion and also goes on to talk about flight control and airplane stability. Later in his life, he proposed the concept of multiplanes (multiple wings mounted on top of each other) and built the first glider triplane named the "boy carrier" in 1849. In fact nearly a century before the first flight of the Wright brothers, Cayley realized both the feasibility and potential of "aerial navigation". Early in his first paper Cayley states that "... in stating the fundamental principles of this art, together with considerable number of facts and practical observations, ... I may be expediting the attainment of an object, that will be found of great importance to mankind; so much so, that a new era in society will commence, from the moment that aerial navigation is familiarly realized". He later goes on to state that "...we shall be able to transport ourselves and families, and their goods and chattels, more securely by air than by water, and with a velocity of 20 to 100 miles per hour" (Cayley 1809-10). So striking has been Cayley's early exposition on aerial navigation that it has led a french aviation historian Charles Dollfus to proclaim in 1923: "L'aéroplane est un invention britannique: il a é té conçu de toutes pièces par George Cayley” (The airplane is a British invention: it has been designed from scratch by George Cayley), and Henry Bouché in 1932: "Sir George Cayley, le véritable inventeur de l'aéroplane" (Sir, George Cayley, the true inventor of the airplane) (Gibbs-Smith 1962). 
While several investigators followed the quest of "aerial navigation" most of Cayley's work seems to have faded into obscurity and appears to be unknown to virtually all aviation enthusiasts in the later half of the nineteenth century. In the years to follow several aeronautical engineers achieved what Anderson describes as "assisted hops" (Anderson 1989). However sustained flight had to wait for the turn of the century. Otto Lilienthal was the first to design and successfully fly controlled gliders in 1891, going on to make over 2500 successful glider flights. Octave Chanute brought aeronautics research to America and designed a biplane glider which directly inspired the designs of the Wright Brothers. Samuel Pierpont Langley was a contemporary of the Wright brothers who built and tested several powered model airplanes. His success in achieving powered flight directly influenced and encouraged the Wright brothers. Just nine days prior to the first flight of the Wright brothers, Langley attempted to launch his powered monoplane however it suffered structural failure. The final historic achievement of successful powered flight was achieved by the Wright brothers. On December $17^{\text {th }}$ 1903, a gasoline powered biplane by the name Wright Flyer I (figure 1.1) took flight in (modern day) Kill Devil Hills, North Carolina, ushering forth the era of practical human flight that was foretold by Sir George Cayley nearly a century ago.

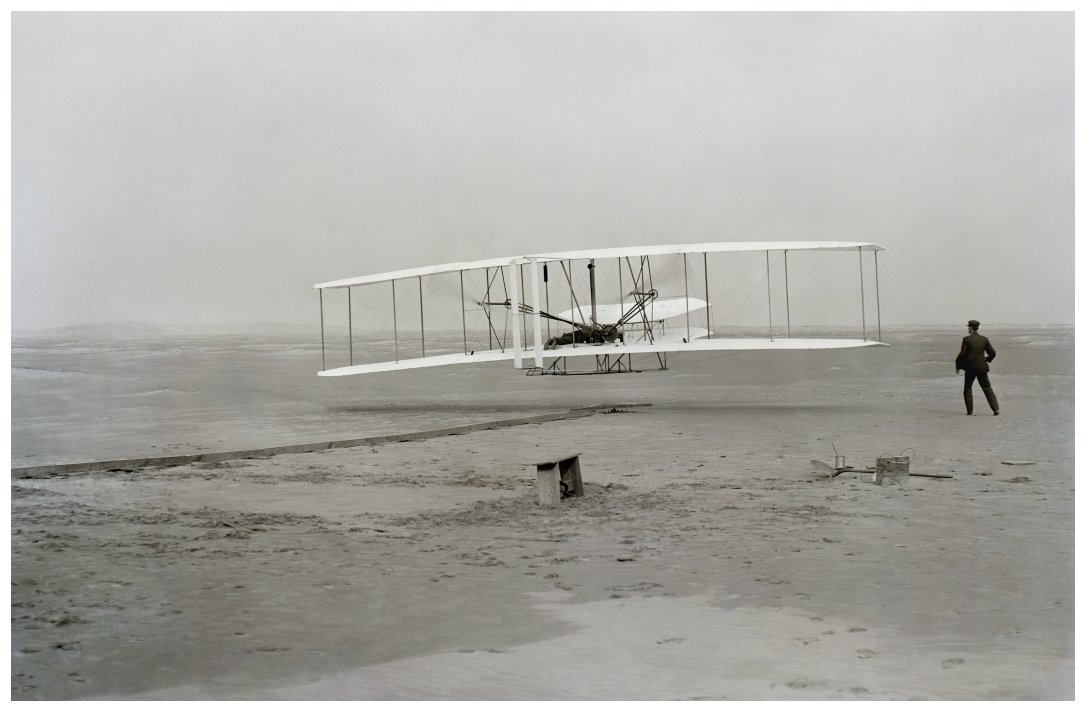

Figure 1.1: First flight of the Wright Flyer I, December 17, 1903, Orville piloting, Wilbur running at wingtip. Image from Wikipedia, the free encyclopedia (2017b).

\subsection{Aeroelasticity and aircraft design}

\subsubsection{Brief history of aeroelasticity}

Since that fateful day, modern airplanes have been used in a variety of different conditions, varying from commercial passenger planes, to supersonic military aircrafts 
(NASA 2014), to endurance flights around the world lasting 9 days (Blakeslee 1986). The myriad uses have resulted in various challenges that needed to be overcome by the aircraft designers. One significant challenge has been due to the dynamic interaction of air flow with the airplane structures, generally studied under the field of aeroelasticity. The failure of Langley's monoplane and the success of the Wright biplane possibly influenced the early designs which ended up being more structurally stable due to the higher rigidity provided by the biplane design (Garrick \& Reed III 1981). However, as the years progressed the maximum speed of the airplanes increased and the designs gravitated towards the monoplane, the aeroelastic problems associated with the interaction of structure and airflow came to the fore. Two of the early cases which saw many fatal failures involved German fighter aircrafts, Albatros D-III, a biplane design, and Fokker D-VIII, a monoplane design. Both of the planes underwent aeroelastic failures associated with static divergence during high-speed dives. The Albatros D-III was used by the German ace Manfred von Richthofen, "The Red Baron", who was among the lucky few to be able land the plane after cracks developed in the lower wing during combat (Garrick \& Reed III 1981). Since during the early years aeroelastic phenomenon was not well understood, monoplanes essentially ceased to exist in the military from 1917 till the mid 1930s (Bisplingoff et al. 1983). However, racer planes continued to be built with the monoplane design with several of them undergoing aeroelastic failure during the 1920s and 1930s (Garrick \& Reed III 1981). Important theoretical progress was made during the late 1920s and 1930s with the works of Glauert (1930); Theodorsen (1935); Karman \& Sears (1938) etc, and mathematical tools which remain relevant to this day were established during this crucial period.

A mention must also be made of the field of wind-turbine design which faces some of the similar issues of aeroelasticity. Some historical development in this area was brought to light by Meijer Drees (1979). Based on his studies of sixteenth and seventeenth century paintings, drawings and engravings of rotors, Meijer Drees (1979) develops a historical account of the evolution of Dutch wind-mill geometry in terms of rotor size and spar location (position of the aeroelastic axis). This is shown in figure 1.2, which shows that the spar location moved from the mid-chord to the quarter-chord position as the size of the rotor increased. Today modern aerodynamic theory tells us that the quarter-chord position is the point of minimum aerodynamic moment on the airfoil. While some of the conclusions of Drees may be conjectural, it appears Dutch wind-mill designers of the seventeenth century may have faced and empirically solved similar aeroelastic problems faced by early aircraft designers of the twentieth century (Shepherd 1990). An overview of the historical development of aeroelasticity can be found in Garrick \& Reed III (1981).

\subsubsection{Modern design challenges}

Today the designers of commercial aircrafts face another challenge brought about by global climate change. With the realization of the contribution of the aviation industry towards climate change (Green 2008), aircraft designers now face a need to significantly improve the fuel efficiency of commercial aircrafts in a bid to reduce the carbon footprint of the industry. In an effort to quantify the opportunities of achieving 


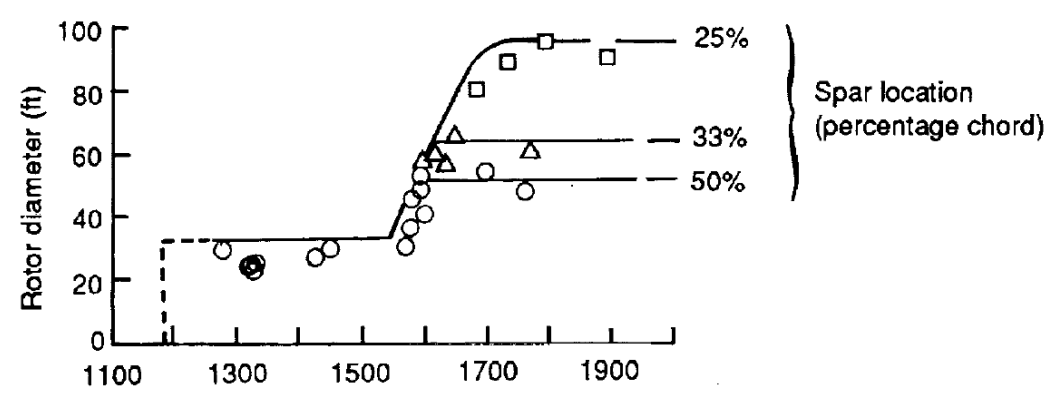

Figure 1.2: Evolution of rotor size and spar location in Dutch wind-mills. Figure is taken from "Historical development of the windmill" Shepherd (1990) and used with permission of NASA.

such an improvement, Schrauf (2005) showed a break-down of the drag experienced by a typical transport aircraft highlighting that frictional drag accounted for more than half the drag experienced by the aircraft. Clearly a favorable modulation of the boundary layer over the wing could help achieve large improvements in fuel efficiency. The modulation could come in the form of effective flow control strategies, or with wing design strategies such as the use of natural laminar flow (NLF) airfoils. Both Schrauf (2005) and Green (2008) push forward the idea that NLF airfoils and laminar flow control strategies are the low-hanging fruits in the goal of higher fuel efficiency and a concerted effort into addressing the engineering challenges for practical implementation must be made.

Some of these challenges require revisiting the aeroelasticity problems from the perspective of laminar wings. As mentioned earlier, pioneering works of Glauert (1930); Theodorsen (1935); Karman \& Sears (1938) etc, provided the insight and modeling of small-amplitude unsteady aerodynamic behavior, and by the 1940s the foundations of unsteady aerodynamics for incompressible attached flows had been laid down. The mathematical framework of these unsteady aerodynamic theories relied on simple inviscid assumptions leading to elegant linear mathematical expressions which proved to be highly attractive to the wing designers (Leishman 2000). Experimental corroboration by Halfman (1952) and Rainey (1957) further added support to the validity of the simple assumptions. Over the next few decades, investigations of unsteady aerodynamics shifted focus to the understanding of unsteady separated flow and the dynamic stall phenomenon, with works of McCroskey et al. (1976); McCroskey (1981); McCroskey et al. (1982); McCroskey (1982); Carr et al. (1977); Crisler et al. (1994). A large body of work on unsteady separated flows was presented by Ericsson \& Reding $(1986,1987,1988 a, b)$. The studies continue to this day with the works of Visbal (2011, 2014); Visbal \& Garmann (2017); Dunne \& McKeon (2015) and several other investigators. A recent review can be found in Coorke \& Thomas (2015). 


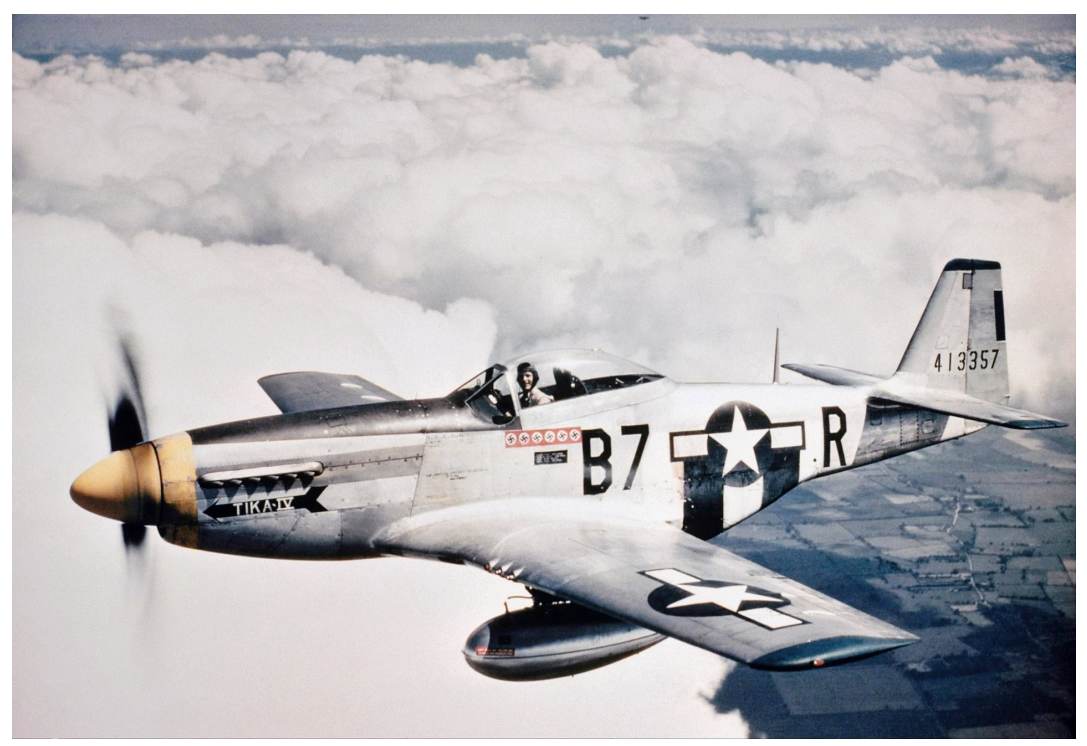

Figure 1.3: North American P-51 Mustang. Image from Wikipedia, the free encyclopedia (2017a).

However it appears that the aeroelasticity problem was not considered from the perspective of natural laminar flow airfoils. This is a surprising fact considering the P-51 Mustang (figure 1.3), a fighter aircraft in the Royal Air Force designed in 1940, incorporated a wing with an NLF airfoil section (Green 2008). The first systematic aeroelastic study on laminar wings appears to have come as late as 2011 through the investigations of Mai \& Hebler (2011). This, along with a subsequent investigation by Hebler et al. (2013), brought to light a peculiar characteristic of unsteady laminar wings, i.e. the presence of non-linearities in the unsteady aerodynamic forces. The classical unsteady aerodynamic theories did not predict non-linear unsteady responses and thus fail to account for such behavior. Inspired by this, Lokatt (2017) performed experiments on unsteady NLF airfoils and also found strong non-linearities in the aerodynamic forces. Consistent in the explanation for the non-linearities in all these studies was the role of transition over the wing surface. These results indicated a need for a more in-depth study of the evolving boundary layer in such unsteady laminar airfoils. Classical theories negate the role of the boundary layer by invoking the inviscid assumption and it is apparent that such an assumption is no longer justified for laminar wings.

While laminar flow technology is aimed at reducing carbon emissions for highspeed commercial aircrafts, the rise of unmanned aerial vehicles (UAVs) have generated interest in the prediction of unsteady aerodynamic characteristics at the low-to-moderate Reynolds number regime. This regime presents a challenging problem from the point of view of aeroelasticity. The elegant linear mathematical theories established in the 
1930s all assume "well-behaved" flow characteristics marked with fully attached and inviscid (high Reynolds numbers) flow. The small length scales and low flight speeds of UAVs make these assumptions questionable. Flow separation is often encountered in the form of a laminar separation bubble which significantly impacts the aerodynamic characteristics. Due to their small size, finite aspect effects can often be important. The review by Mueller \& DeLaurier (2003) highlights some of the aerodynamic characteristics of UAVs. In the review the authors note that that unsteady aerodynamic investigations in this regime have only recently become of interest and that no studies exist on the gust response for small UAVs. Recent experimental and numerical works of Poirel et al. (2008); Poirel \& Yuan (2010); Poirel \& Mendes (2014); Barnes \& Visbal $(2016,2018)$ on the aeroelasticity of wings at transitional Reynolds numbers have highlighted the failure of the classical theories in this regime. The studies again point to the key role played by laminar separation during the unsteady dynamics.

\subsection{Boundary layers over a stationary wing}

While NLF airfoils have been proposed as one of the key engineering solutions for drag reduction, laminar flow at high Reynolds numbers is susceptible to destabilization and may not always be possible. Thus turbulent drag reduction strategies need to be used effectively where needed (Bushnell 2003). While the understanding and prediction of unsteady boundary layers presents an arduous challenge, the investigations of turbulent boundary layers evolving over a stationary wing is by no means complete. The understanding of the structure and scaling of wall-bounded turbulent flows has been in study for several decades and the investigations are still ongoing. These flows have been studied with different canonical geometries such as channels (Kim et al. 1987; Moser et al. 1999; Lee \& Moser 2015), pipes (El Khoury et al. 2013; Jiménez \& Hoyas 2008; Chin et al. 2015) and flat plates Spalart (1988); Schlatter \& Örlü (2010); Eitel-Amor et al. (2014). For the case of spatially evolving boundary layers over a flat plate, the simplest canonical case involves boundary-layer evolution subjected to a zero pressure gradient (ZPG). These flows may be uniquely characterized by a single parameter, i.e. the Reynolds number Re. However, practical flow cases are often influenced by pressure gradients, which leads to another class of boundary layers known as "equilibrium boundary layers" that maintain a constant ratio of viscous and pressure forces throughout their development. These boundary layers are characterized by two parameters - Re and the pressure gradient parameter $\beta$ (Clauser 1954). The developing boundary layer over an airfoil however further increases in complexity since these boundary layers fall under the category of non-equilibrium boundary layers where the Clauser parameter is spatially varying. In such cases the flow history also plays a role in determining the local boundary-layer properties (Clauser 1954; Bobke et al. 2017). Analysis of such flow cases becomes significantly more difficult since the local boundary-layer parameters do not uniquely define the state of the boundary layer. Nonetheless the study of such boundary layers is important since generic boundary layers found in nature would belong to this "non-equilibrium" category, which includes the boundary layers over wings.

In light of the various aerodynamic design challenges, the focus of the current thesis is on the investigation of boundary layers developing over wings in various 
different flow regimes. The primary focus has been on the investigation of unsteady scenarios in the context of aeroelastic stability, specifically in flow regimes where the classical unsteady aerodynamic theories have been found to be unreliable.

The thesis is structured as follows. A brief introduction of the numerical methods used for the simulations is given in Chapter 2. An overview of the key results obtained during the course of the work is presented in Chapter 3. The main conclusions of the current work are provided in Chapter 4 along with an outlook for future investigations. The second part of the thesis contains the individual papers and technical reports. 


\section{Numerical Method}

\subsection{Numerical Discretization}

The numerical code used for the simulations is Nek5000, which is an open source research code developed by Fischer et al. (2008) at Argonne National Laboratory. The code solves the incompressible Navier-Stokes equations (2.1) in non-dimensional form:

$$
\begin{aligned}
\frac{\partial u_{i}}{\partial t}+u_{j} \frac{\partial u_{i}}{\partial x_{j}}=-\frac{1}{\rho} \frac{\partial p}{\partial x_{i}}+\frac{1}{R e}\left(\frac{\partial^{2} u_{i}}{\partial x_{j} \partial x_{j}}\right) & +f_{i} \\
\frac{\partial u_{i}}{\partial x_{i}} & =0
\end{aligned}
$$

where $x_{i}$ is the coordinate direction, $u_{i}$ is the velocity component, $p$ is the pressure, $f_{i}$ is the volume forces acting on the fluid, and $R e$ is the defined Reynolds number, with all variables being in their non-dimensional form. The non-dimensionalization of the variables is performed with a certain velocity scale $U^{*}$, length scale $l^{*}$ and reference density $\rho^{*}$, where the superscript * represents dimensional quantities. The Reynolds number is then defined as $\operatorname{Re}=\rho^{*} U^{*} l^{*} / \mu^{*}$, where $\mu^{*}$ is the dynamic viscosity. The solution of the Navier-Stokes is found for its weak form with a spectral-element method, first proposed by Patera (1984). The method allows the mapping of elements to complex geometries along with a high-order spatial discretization within the elements, thus combining the generality of finite-element methods with the accuracy of spectral methods (Patera 1984). The spatial discretization in each element is performed following the $\mathbb{P}_{n}-\mathbb{P}_{n-2}$ (Maday \& Patera 1989) formulation with the velocity represented by $n^{\text {th }}$ order Lagrange interpolants through the Gauss-Lobatto-Legendre (GLL) quadrature points, while the pressure is represented with $(n-2)^{\text {th }}$ order interpolants through the staggered Gauss-Legendre (GL) quadrature points. The nonlinear terms are treated explicitly by third-order extrapolation (EXT3) and over-integration is used for the removal of aliasing errors arising out of the evaluation of the non-linear term. The viscous terms are treated implicitly and time integration is performed using a third-order backward differentiation (BDF3). The divergence constraint is enforced using a third-order accurate pressure correction scheme (Heinrichs 1996). Nek5000 is written in Fortran 77 and $\mathrm{C}$ with efficient scaling for up to 1 million MPI ranks (Fischer et al. 2015). Further details of the numerical method can be found in Deville et al. (2002). 


\subsection{Relaxation-term large-eddy simulation (RT-LES)}

Owing to the high Reynolds numbers and large time-scales of integration for some of the flow cases, a direct numerical simulation (DNS), which requires a resolution of all the spatial scales of the flow, leads to prohibitively high computational costs. The technique of wall-resolved large-eddy simulation (LES) provides a computationally cheaper alternative to DNS, while also exhibiting the high-fidelity characteristics of DNS. In recent years the technique has been successfully utilized in the investigations of spatially developing boundary layers (Eitel-Amor et al. 2014), pipe flows (Chin et al. 2015) and flow over wings (Uzun \& Hussaini 2010; Lombard et al. 2016). The success of the approach has motivated its use in the present work. The wall-resolved LES method used is based on the three-dimensional relaxation term (RT3D) variant of the approximate deconvolution with relaxation term (ADM-RT) approach first used by Schlatter et al. (2004). The method has been shown to be reliable in accurately predicting transition and also preserving the characteristic structures of transitional flows (Schlatter et al. 2006b) which are observed in the DNS investigations. This particular quality of the LES model is crucial since there is a large focus on the unsteady transition in the present work. The LES method supplements the governing equations with a dissipative term $-\chi \mathcal{H}(u)$. The equations of motion for the resolved velocity and pressure thus read as

$$
\begin{array}{r}
\frac{\partial u_{i}}{\partial t}+u_{j} \frac{\partial u_{i}}{\partial x_{j}}=-\frac{1}{\rho} \frac{\partial p}{\partial x_{i}}+\frac{1}{R e}\left(\frac{\partial^{2} u_{i}}{\partial x_{j} \partial x_{j}}\right)+f_{i}-\chi \mathcal{H}\left(u_{i}\right), \\
\frac{\partial u_{i}}{\partial x_{i}}=0,
\end{array}
$$

where $\mathcal{H}$ is a defined high-pass spectral filter and $\chi$ is a model parameter which together with $\mathcal{H}$ determines the strength of the dissipative term. The high-pass filter function $\mathcal{H}$ is defined such that the resultant relaxation-term only has energy in the highest modes, defined by a cut-off mode-number $N_{c}$. Figure 2.1 illustrates the shape of the filter function in spectral space.

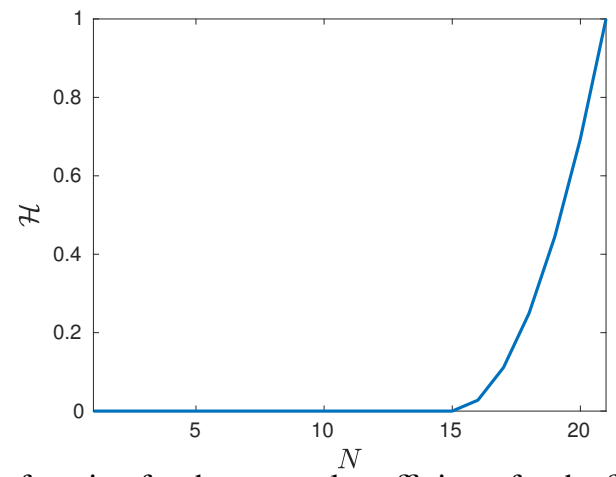

Figure 2.1: Transfer function for the spectral coefficients for the filter $\mathcal{H}$ with number of modes $N=21$ and cut-off mode number $N_{c}=16$. 
Several parameter optimization studies were performed to determine the optimum value of $\chi$ and filter shape $\mathcal{H}$ using turbulent channel flow simulations. The LES results were compared with the DNS database of Moser et al. (1999) and the optimum parameters were further validated for a flow around a wing section at $R e_{c}=400,000$. A good agreement was found between the LES and the DNS data of Hosseini et al. (2016), and the optimized parameters were then used for all subsequent LES. The validations of the turbulent kinetic energy (tke) budget for a channel flow at $R e_{\tau}=395$ and the wing section at $R e_{c}=400,000$ are shown in figure 2.2
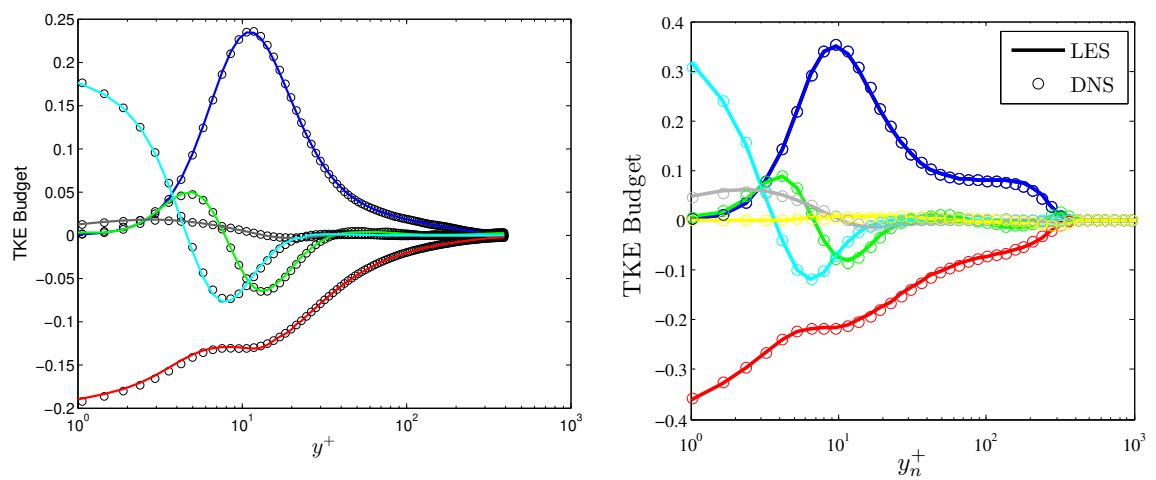

Figure 2.2: (Left) comparison of the tke budget for a channel flow case with the DNS data of Moser et al. (1999). (Right) Comparison of the tke budget with DNS data of Hosseini et al. (2016) for flow over a wing section at $R e_{c}=400,000$. All values are normalized with inner units. The individual terms are color coded as: Production (blue), dissipation (red), viscous diffusion (cyan), turbulent diffusion (green), and velocity-pressure correlation (gray).

\subsection{Arbitrary-Lagrangian-Eulerian (ALE)}

Typical solutions of unsteady fluid flows utilize the Eulerian framework where the coordinate system is fixed in space. However difficulties arise with a fixed co-ordinate system when the domain boundaries are in motion, as is the case of fluid-structure interaction problems, or when there is a free surface which may lead to a deforming interface. An appropriate method is needed to account or the motion of the boundaries and/or the interior grid points. One such method which significantly simplifies the difficulties arising out of moving boundaries is the Arbitrary-Lagrangian-Eulerian (ALE) method. The method was proposed in a finite-difference framework by Hirt et al. (1974) and later brought to the spectral-element framework by Ho \& Patera $(1990,1991)$. The technique combines both the Lagrangian and Eulerian formulations such that, the Navier-Stokes may be solved with the grid points moving with the fluid elements (Lagrangian framework), or with fixed grid points (Eulerian framework), or with grid points moving in an arbitrary prescribed manner. The heart of the technique lies in the formulation of the total time rate of change of a quantity $F$, along a point moving with 
a velocity $\mathbf{w}$, defined analogously to the material derivative. The expression is referred to as the ALE derivative (Deville et al. 2002), here denoted as $\partial F /\left.\partial t\right|_{\mathrm{w}}$ to differentiate it from the very similar expression for the material derivative (which is evaluated along the fluid particle trajectory)

$$
\left.\frac{\partial F}{\partial t}\right|_{\mathbf{w}}=\frac{\partial F}{\partial t}+w_{i} \frac{\partial F}{\partial x_{i}} .
$$

When $w_{i}$ is equal to the fluid velocity $u_{i}$, we recover the familiar Lagrangian expression for the material derivative $D F / D t$. On the other hand, when $w_{i}=0$, we get the local (Eulerian) rate of change of the quantity $F$. The material derivative and the ALE derivative share a simple relationship defined using a relative velocity of the fluid particle with respect to the grid motion $c_{i}=u_{i}-w_{i}$, which may be used in the definition of material derivative to obtain

$$
\frac{D F}{D t}=\left.\frac{\partial F}{\partial t}\right|_{\mathbf{w}}+c_{i} \frac{\partial F}{\partial x_{i}} .
$$

Thus the Navier-Stokes in the ALE formulation may be expressed as

$$
\begin{aligned}
\left.\frac{\partial u_{i}}{\partial t}\right|_{\mathbf{w}}+\left(u_{j}-w_{j}\right) \frac{\partial u_{i}}{\partial x_{j}}=-\frac{1}{\rho} \frac{\partial p}{\partial x_{i}}+\frac{1}{R e}\left(\frac{\partial^{2} u_{i}}{\partial x_{j} \partial x_{j}}\right) & +f_{i}, \\
\frac{\partial u_{i}}{\partial x_{i}} & =0,
\end{aligned}
$$

where $\mathbf{w}$ is the velocity of the grid points. The solution of the Navier-Stokes is then a simple matter of specifying a suitable grid velocity.

In many cases, such as the flow over an oscillating airfoil, the velocity of the grid points at the boundary (airfoil surface) may be explicitly known. Ho \& Patera $(1990,1991)$ propose to extend this velocity to the interior points of the domain by solving an elliptic problem for the mesh velocity. In the present work we take a simpler approach to prescribing the mesh velocities in the interior domain. Recognizing the simple trigonometric form of a harmonic pitching motion, all mesh points may simply be prescribed a solid body rotation with the instantaneous angular velocity of the airfoil. However a pure solid-body rotation would also displace the domain boundaries. Therefore a damping function is used to smoothly reduce the rotational velocity away from the airfoil boundary such that the mesh motion is zero at the far-field, inlet and outflow boundaries. For an airfoil with an instantaneous rotation rate of $\Omega_{z}(t)$, the mesh velocity may be prescribed as:

$$
w(x, y, z, t)=\underbrace{\left(\Omega_{z}(t) \times R\right)}_{\text {Solid body rotation }} \overbrace{f(x, y, z)}^{\text {Damping }},
$$

where $R$ is the distance of the point from the rotational axis and $f$ is a damping function which can be prescribed in many different ways, depending on one's preferences. The damping function needs to have two essential properties, i.e. it must be equal to 1 at the moving boundary, which implies the mesh points at the interface boundary move with the surface (solid body rotation at the airfoil surface), and it must smoothly decay to zero close to the far-field boundaries, which allows the external boundaries of the 
computational domain to remain fixed in physical space. Figure 2.3 shows the damping function used in the present work as a function of the normal distance from the airfoil surface. The damping function moves the grid points close to the airfoil surface with

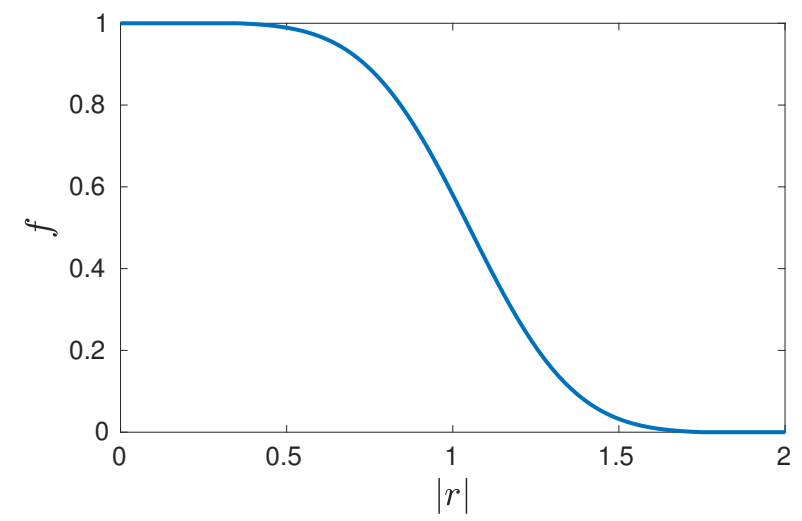

Figure 2.3: Damping function $f$ for the mesh velocities as a function of distance from the surface $r$.

the same rotational velocity of the airfoil and spreads out the mesh deformation into the interior of the domain. This damping function is calculated once at the beginning of the simulation. Hence all quantities $\Omega_{z}(t), \vec{r}, f(r)$ which are needed for prescribing the mesh velocity are explicitly known at each time-step without the need for solving an elliptic equation as in Ho \& Patera $(1990,1991)$.

\subsection{Fluid-Structure coupling}

The fluid-structure-interaction problems considered within the current work fall into two classes according to the coupling between the individual systems. One-way coupled problems, which represent the class of problems where the time-dependent structural motion is known apriori. Coupling between the two systems is fairly trivial since the structure does not react to fluid forces and the velocity boundary conditions for the fluid are determined from the (known) velocity of the structure. Forced oscillation of a pitching airfoil falls into this category.

The second class is that of two-way coupled problems where the fluid forces influence the structural motion. For the partitioned time marching algorithms, the coupling between the two systems is non trivial since both systems affect the dynamic behavior of each other. In the current work the fluid-structure coupling is performed using the Green's function approach recently developed by Fischer $e$ t al. (2017). It is a semi-implicit technique which allows for a fully-implicit treatment of the added-mass effects while explicitly treating the geometric non-linearities. The approach builds on the results of Causin et al. (2005) where the authors identified the added-mass effects as a linear phenomenon. Fischer et al. (2017) incorporate the added-mass effects into the solution of the coupled problem through a Stokes update step. Unlike a fully implicit 
method like fixed-point iterations with relaxation (Küttler \& Wall 2008), the Green's function approach is iteration free at the cost of an added Stokes solve. The method can easily be extended to structures where multiple degrees of freedom of the structure are active, with each active degree of freedom requiring a Stokes solve. The method has been tested even for a zero mass solid and presents no numerical difficulties (Fischer et al. 2017). The validation of the implementation is done for an oscillating cylinder at $R e=100$ with a solid to fluid density ratio of $\rho_{s} / \rho_{f}=10$. The cylinder motion is modeled using a spring-mass system with a spring natural frequency of $f=0.167$. The same case considered in Fischer et al. (2017) and also in the works of Prasanth \& Mittal (2008). Figure 2.4 shows the time variation of the vertical position of the oscillating cylinder. The limit cycle oscillations stabilized with an amplitude of $\eta_{\max }=0.508$ and angular frequency of $\omega=1.04$, which are within $1 \%$ of the values reported in Fischer et al. (2017) and Prasanth \& Mittal (2008). In addition, the fully implicit fixed-point iteration method described in Küttler \& Wall (2008) has also been implemented. The results of the two methods are practically identical for all tested cases.

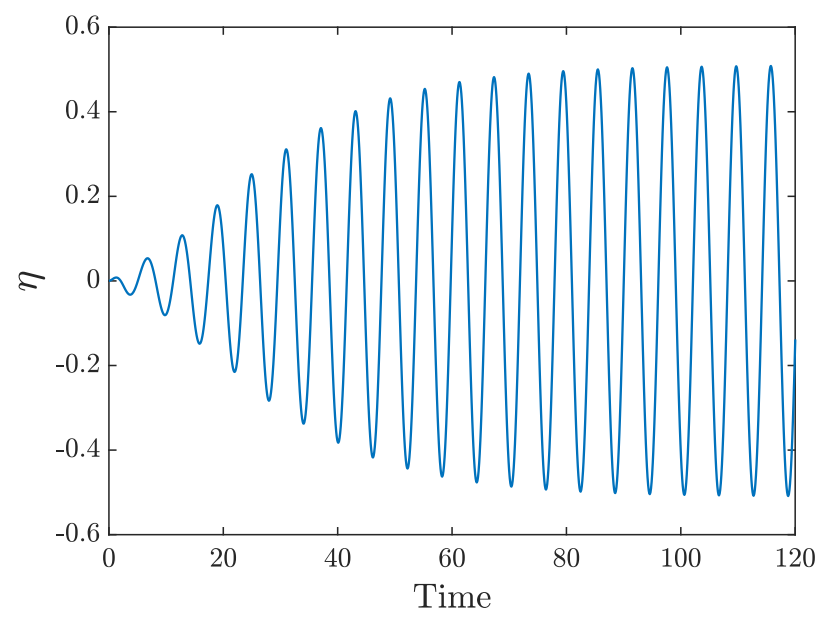

Figure 2.4: Temporal variation of the vertical position of an oscillating cylinder at $R e=100$.

\subsection{Free-stream turbulence}

In several of the numerical studies in the current work, isotropic, homogeneous freestream turbulence is prescribed at the inlet and far-field boundaries to add small disturbances to the flow-field, which simulate the disturbances found in a wind-tunnel or in free-flight conditions. The free-stream turbulence is prescribed as a superposition of Fourier modes with a random phase shift. The maximum and minimum amplitudes of the wavenumber vector are prescribed quantities and are limited by the resolution of the spatial discretization and size of the domain respectively. The wavenumber space between the minimum and the maximum is divided into 20 concentric shells with 
each shell representing the amplitude of the three-dimensional wavenumber vectors lying on the shell. 20 points are randomly chosen on each shell with the location of each point representing the three-dimensional components of the wavenumber vector. Thus the free-stream turbulence is represented by a total of 400 fourier modes. Care is taken to avoid very small wavenumber components which result in wavelengths in physical space that are larger than the computational domain. The streamwise length scales are transformed to a temporal frequency by invoking Taylor's frozen turbulence hypothesis and using the local mean streamwise velocity at the inlet for the space-time conversion. The amplitude of the free-stream modes on each spherical shell is scaled using the von Kármán spectrum. Figure 2.5a shows an instantaneous visualization of the streamwise velocities in a doubly periodic duct flow case with high (5\%) freestream turbulence intensity prescribed at the the inlet. Figure $2.5 \mathrm{~b}$ shows the spatial decay of turbulence intensity. After a small initial distance of adjustment from the inlet, the turbulence intensity decays as a power law. A very similar method for generating free-stream turbulence for simulations of flat-plate boundary layers is used by Schlatter (2001); Brandt et al. (2004); Schlatter et al. (2008) and more recently for wind turbine simulations by Kleusberg (2017).

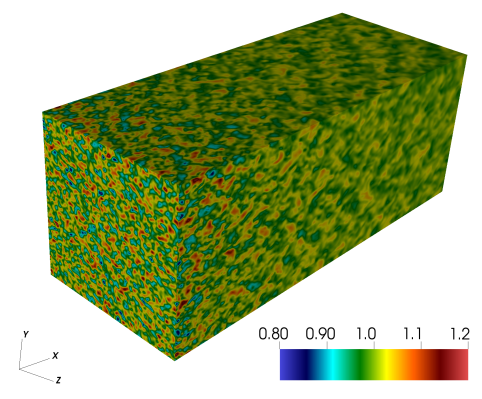

(a)

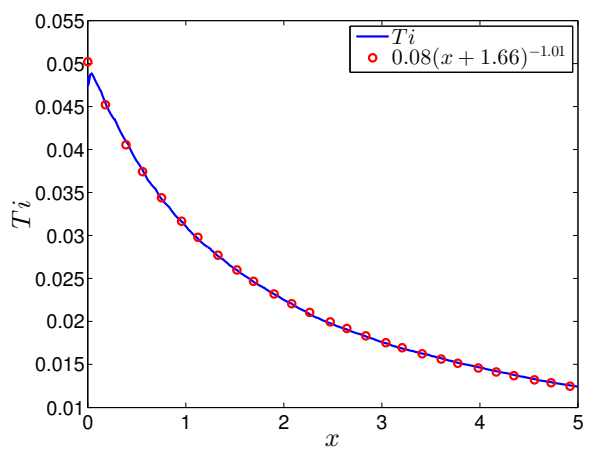

(b)

Figure 2.5: (a) Visualization of free-stream turbulence prescribed at the inlet for a doubly periodic duct flow. Colors represent the instantaneous streamwise velocity. (b) Decay of turbulence intensity with streamwise distance, along with the leastsquares fit of a power law. 
CHAPTER 3

\section{Overview of results}

\subsection{Introduction}

In this section the key results of the thesis are covered. The main focus of the work has been on the investigation of unsteady wings, with the studies ranging from linear stability analysis to the analysis of unsteady transition. An overview of the results from the studies on unsteady wings (Paper 2-5) is presented in this section. In addition, the thesis contains two other articles. The first of these two (Paper 1) is an investigation of the stability properties of the spectral-element method (SEM) and the effects of the commonly used "evolve and filter" procedure used for the stabilization of the method. The other article (Paper 6) investigates the evolution of a turbulent boundary over a stationary wing section across different Reynolds numbers. These two articles are not covered in the overview and the reader is referred to the individual papers for the details.

\subsection{Linear stability of fluid-structure-interaction problems}

Paper 2 describes the development of a formulation for the linear stability analysis of fluid-structure-interaction (FSI) problems. FSI problems span a diverse range of topics - fluttering of flags (Shelley \& Zhang 2011), phonation (Heil \& Hazel 2011), blood flow (Freund 2014), motion of bubbles (Ern et al. 2012), aircraft stability (Dowell \& Hall 2001), vortex induced vibrations (Williamson \& Govardhan 2004) etc. The phenomena that emerge out of an FSI problem are often highly non-linear, dynamically rich and can exhibit different flow regimes such as fluttering and tumbling of plates falling under gravity (Mittal et al. 2004). Despite strong non-linearities, the linear analysis of systems can often provide valuable insights into the dynamical behavior of systems. In particular, state transitions may often be governed by a linear mechanism. However, for FSI linear analysis also presents a computational challenge in that moving boundaries need to be treated. In the context of parallel flows the challenge was addressed by Benjamin (1959, 1960) and Landahl (1962). Their theoretical treatment of moving boundaries found widespread application in compliant wall problems (Riley et al. 1988). The treatment of slightly more complex problems with rigid-body motion in a non-inertial frame started with the global stability analysis of an oscillating cylinder by Cossu \& Morino (2000). Several other investigations of rigid-body type motion in a non-inertial reference frame have followed (Mittal \& Singh 2005; Fabre et al. 2011; Tchoufag et al. 2014a,b; Navrose \& Mittal 2016; Cano-Lozano et al. 2016). 
Rigid-body motion and compliant walls form the bulk of the linear investigations in FSI. There are a few notable exceptions to this. Fanion et al. (2000) have used a transpiration approach to arrive at a more general linear formulation for the weak form of the Navier-Stokes. This has been used by Fernández \& Le Tallec $(2003 a, b)$. Goza et al. (2018) have performed linear FSI on an inverted flapping flag using the immersed boundary method and, in very contemporary work Pfister et al. (2019) have advocated an ALE based formulation for linear FSI. In the current work we have followed what is in essence the method used for compliant walls and is also the same approach used in the weak formulation of Fanion et al. (2000). However, we approach the problem in its strong form and arrive at a slightly different formulation from the one obtained by Fanion et al. (2000).

The statement of a linear stability problem is that, given a stationary base state defined by $\left(\mathbf{U}^{0}, P^{0}, \boldsymbol{\eta}^{0}\right)$, one needs to find the evolution equations for small-amplitude perturbations $\left(\mathbf{u}^{\prime}, p^{\prime}, \boldsymbol{\eta}^{\prime}\right)$, such that to a first-order approximation the perturbed state may be given by a superposition of the base state and small-amplitude perturbations. Here $\mathbf{U}$ is the fluid velocity, $P$ is the pressure and $\boldsymbol{\eta}$ is the structural variable (usually its position). Variables with superscript ${ }^{0}$ denote base state quantities while variables with superscript ' denote perturbation quantities. Bold symbols indicate vectors. From a numerical point of view, one must also specify that the base state is defined on the equilibrium grid $\mathbf{x}^{0}$ and the perturbations evolve on a time varying grid $\mathbf{x}$. Therein lies the heart of the problem for linear FSI. Since for an FSI problem the grid points move due to a moving interface, as for example is the case in an ALE based approach, one needs to treat two quantities - the base state defined on an equilibrium grid and the perturbed state on a moving grid, in a consistent manner. This has been resolved in the current work by defining both the base state as well as the perturbed state on the equilibrium grid, and using a first-order Taylor expansion of the base flow and perturbations to evaluate the quantities on the perturbed grid. A schematic of the approach is shown in figure 3.1. The blue lines represent the equilibrium grid while the red lines indicate the perturbed position of the grid. In addition to the variable definitions, one also requires the treatment of derivatives evaluated at the perturbed grid based on quantities defined on the equilibrium grid. This requires a mapping of the coordinates, which in the small-amplitude limit has been assumed to be linear and of the form $\mathbf{x}=\left[\mathcal{I}+\mathcal{R}^{\prime}\right] \mathbf{x}^{0}$. For $|\mathcal{R}| \ll 1$, the inverse mapping may be conveniently expressed as $\left[I+\mathcal{R}^{\prime}\right]^{-1} \approx\left[I-\mathcal{R}^{\prime}\right]$, and may be used in the evaluation of derivatives at the perturbed position. We refer to this as the geometric linearization of the problem. The Navier-Stokes may now be consistently evaluated at the perturbed positions using the Taylor-expanded terms and the geometric linearization. The resulting expression ends up having a large number of terms which would need to be simplified. Should one persist with the full expansion and simplification, one is rewarded with the following 


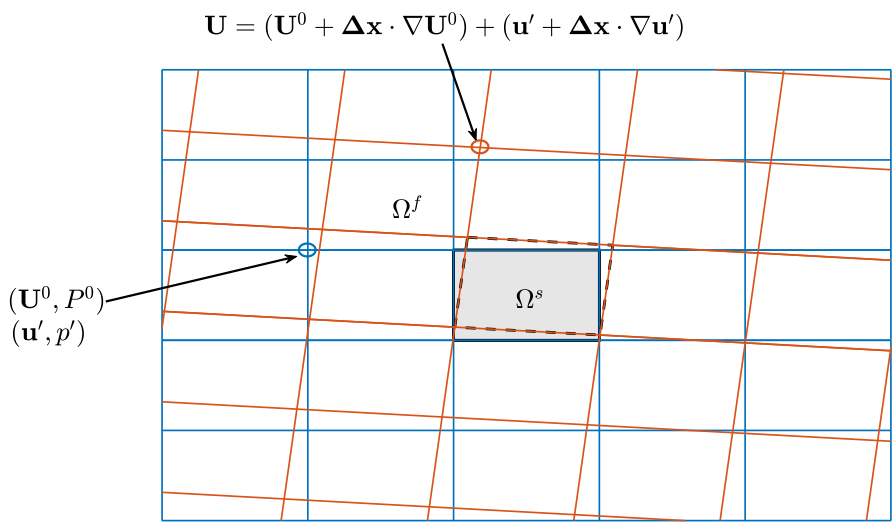

Figure 3.1: Schematic of the treatment of fluid variables in a moving domain.

compact final expression

$$
\begin{aligned}
& {\left[U_{j}^{0} \frac{\partial U_{i}^{0}}{\partial x_{j}^{0}}+\frac{\partial P^{0}}{\partial x_{i}^{0}}-\frac{1}{R e} \frac{\partial^{2} U_{i}^{0}}{\partial x_{j}^{0} \partial x_{j}^{0}}\right]+\Delta x_{l} \frac{\partial}{\partial x_{l}^{0}}\left[U_{j}^{0} \frac{\partial U_{i}^{0}}{\partial x_{j}^{0}}+\frac{\partial P^{0}}{\partial x_{i}^{0}}-\frac{1}{R e} \frac{\partial^{2} U_{i}^{0}}{\partial x_{j}^{0} \partial x_{j}^{0}}\right]} \\
& +\left[\frac{\partial u_{i}^{\prime}}{\partial t}+U_{j}^{0} \frac{\partial u_{i}^{\prime}}{\partial x_{j}^{0}}+u_{j}^{\prime} \frac{\partial U_{i}^{0}}{\partial x_{j}^{0}}+\frac{\partial p^{\prime}}{\partial x_{i}^{0}}-\frac{1}{R e} \frac{\partial^{2} u_{i}^{\prime}}{\partial x_{j}^{0} \partial x_{j}^{0}}\right]=0,
\end{aligned}
$$

with all terms and derivatives now evaluated on the equilibrium grid (denoted with the superscript ${ }^{0}$ for all the partial derivatives). The terms in the first two brackets of the expression may be identified as the first-order Taylor expansion of the steady base flow, representing the evaluation of the base flow at the perturbed points. These completely drop out of the perturbation equations in a first-order approximation. The terms of the final bracket are simply the standard terms of the linear Navier-Stokes. Hence the linear form of the equations for an FSI problem is no different from the case with no moving boundaries. There arises one exception when satisfying the no-slip condition at the perturbed boundary, where the Taylor expansion of the base flow must also be taken into account. Therefore the no-slip boundary condition at the FSI interface changes to a transpiration boundary condition

$$
u_{i}^{\prime}=\frac{d\left(\Delta x_{i}\right)}{d t}-\left(\Delta x_{j} \frac{\partial U_{i}^{0}}{\partial x_{j}^{0}}\right) .
$$

A global balance of the fluxes further shows that the only additional forces at the perturbed boundary arise due to the fluid perturbations. This is in contrast to the formulation of Fanion et al. (2000) where the authors claim boundary stresses due to the base flow at the new position must also contribute to the balance of the stresses acting on the structure. Fernández \& Le Tallec $(2003 a, b)$ have referred to these 
additional stresses as "added stiffness" terms. In the current work these terms are shown to always vanish at first-order. The mathematical derivation is followed by numerical tests for validation and we find that the "added stiffness" does not play any role in the linearized dynamics.

Due to the limitations of the numerical solver (and the scope of the current thesis work) we have limited the study to rigid-body motion problems where, the structural equations are modeled using a spring-mass-damper system described by

$$
\mathcal{M} \frac{\partial^{2} \eta_{i}}{\partial t^{2}}+\mathcal{D} \frac{\partial \eta_{i}}{\partial t}+\mathcal{K} \eta_{i}=\mathcal{F}_{i},
$$

where $\mathcal{M}$ represents the system inertia, $\mathcal{D}$ represents the damping, $\mathcal{K}$ represents the spring stiffness, and $\mathcal{F}$ represents the fluid forces. $\eta$ represents the structural variable described earlier. Several test cases have been considered for the validation. The structural equations are modeled using a spring-mass-damper system and low solid-tofluid density ratios (in the range of $1-10$ ) have been considered so as to encourage significant structural response in the FSI system. The validation performed for a rotating ellipse at $R e=50$, initially held at an angle to the oncoming flow is shown in figure 3.2. No spring stiffness and damping is considered, hence the only acting forces on the ellipse are those due to the fluid. The case is considered to encompass the general characteristics for rigid-body motion since the base state is calculated when the ellipse is rotated by an angle of $30^{\circ}$ and thus the flow contains no inherent symmetries. The rotational displacement of the FSI surface causes both displacement as well as change in the direction of the normals at the boundary. The linear and non-linear evolutions have been compared for identical small-amplitude disturbances and the agreement between the two is found to be extremely good. The peak amplitudes of the individual oscillations in figure 3.2a trace a linear line in a logscale signifying the exponential growth of instabilities (figure 3.2b), which is well captured by both the linear and non-linear simulations. The difference in growth rate and frequencies obtained from the linear and nonlinear simulations is less than $0.1 \%$ in all test cases. Even transient behavior of the system is well captured by the derived linear system as shown in figure 3.2a.

The formulation is then applied to the case of a rotating cylinder with an attached rigid splitter-plate aligned with the streamwise direction of the flow. This particular case along with a similar one with a flexible splitter-plate has enjoyed attention in the recent years due to its peculiar characteristic of undergoing a symmetry breaking bifurcation wherein, the splitter-plate rotates and aligns itself at a non-zero angle to the flow (Xu et al. 1990; Bagheri et al. 2012; Lācis et al. 2014). This occurs at both sub-critical and super-critical Reynolds numbers. An eigenvalue analysis of the system at two different Reynolds numbers ( $R e=45 \& 156)$ using the linear FSI equations reveals that the onset of symmetry breaking is due to a zero frequency unstable mode (figure 3.3). In the aerodynamic literature this is known as a divergence instability. This mode is identified as the cause of symmetry breaking since it leads to a continuous rotation of the cylinder. For the final symmetry breaking to take place a non-linear mechanism is required since the (non-linear) flow equations need to equilibrate at the 


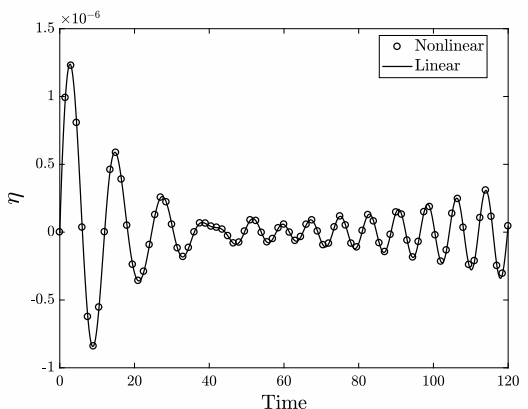

(a)

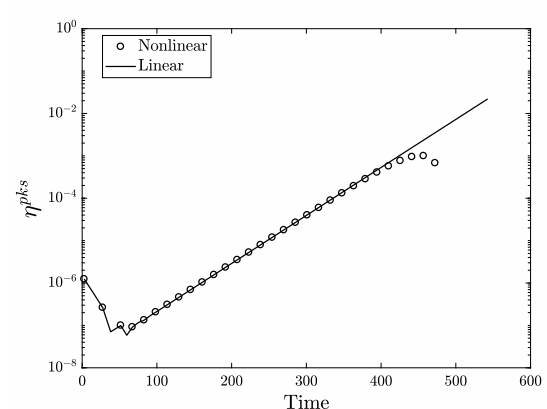

(b)

Figure 3.2: Comparison of linear and non-linear evolution of the position of a rotated ellipse at $R e=50$. (a) Initial time evolution of rotational angle $\eta$. (b) Evolution of peak amplitudes of the rotational angle in a semi-log scale.

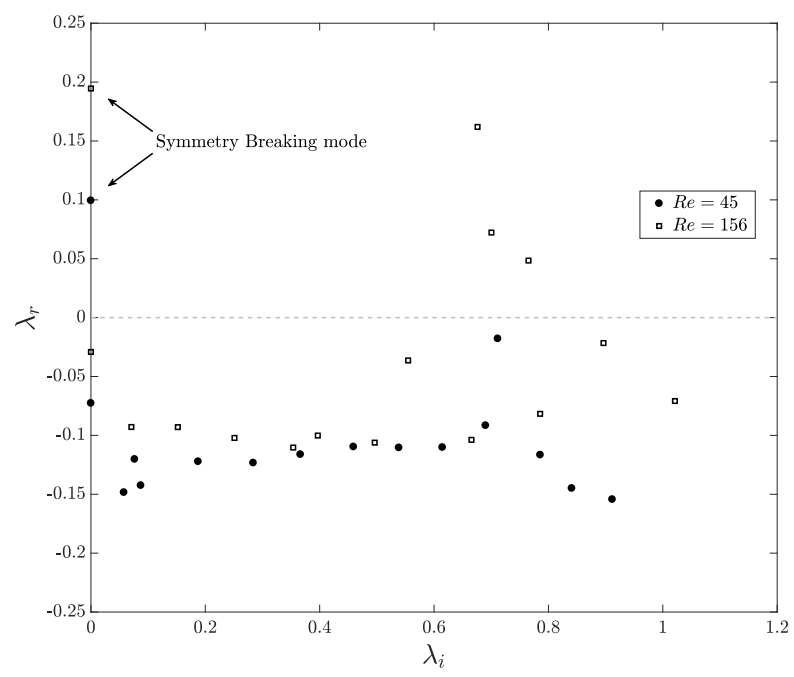

Figure 3.3: One-sided spectra for the rotating cylinder with a rigid splitter plate that exhibits spontaneous symmetry breaking at $R e=45$ and $R e=156$.

new angle. However the onset itself can be identified to this zero frequency unstable mode.

Finally, the structural sensitivity of the eigenvalue is performed through the adjoint equations for an oscillating cylinder at $R e=50$ to investigate how the introduction of a structural degree of freedom changes the system sensitivity. A comparison is made with the now classic picture of the eigenvalue sensitivity of a stationary cylinder reported 

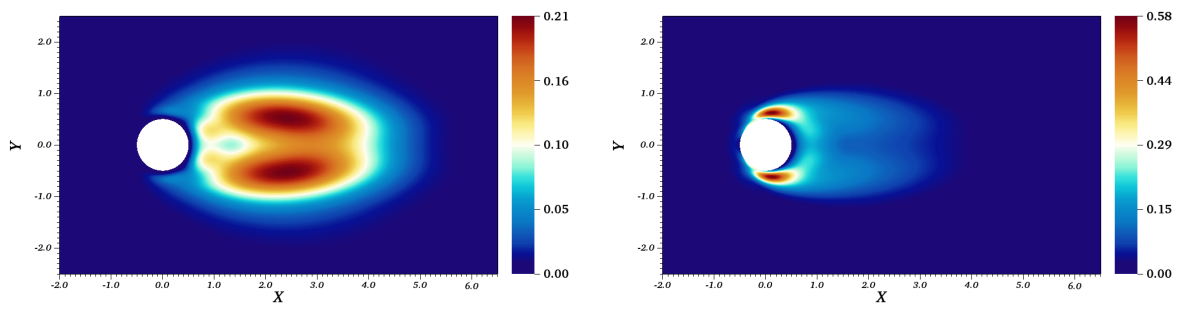

Figure 3.4: Comparison of the structural sensitivity map for the least stable eigenvalue for a (left) stationary cylinder and (right) for the FSI system when the structural natural frequency is close to the unstable frequency.

by Gianetti \& Luchini (2007). The sensitivity is studied by parametrically varying the natural frequency of the spring-mass system $\left(\omega_{n}=\sqrt{\mathcal{K} / \mathcal{M}}\right)$ and evaluating the sensitivity of the unstable eigenvalue. Figure 3.4 shows a comparison of the stationary case, with the case when the structural eigenvalue is close to the unstable eigenvalue of the coupled FSI system. The sensitivity map changes drastically as compared to the stationary system. While for the stationary system the dominant region of sensitivity lies in the near wake, the dominant region shifts much closer to the structure for the FSI system close to resonance.

From the perspective of the fluid variables, the derivation is completely general and makes no assumptions on how the FSI interface deforms. This deformation is governed by the type of structural modeling involved in the FSI problem, which would of course also need to be linearized. All information regarding the interface deformation is contained within the variable $\Delta \mathbf{x}$ in equation 3.2 at the FSI interface. However the scope of the structural equation (and validations) in the current work is confined to rigid-body motion.

\subsection{Onset of pitch-oscillations at transitional Reynolds numbers}

The aeroelastic investigation of Poirel et al. (2008) is possibly the first systematic study on the aeroelasticity of wings at transitional $R e$. The authors conducted several aeroelastic tests on a NACA0012 airfoil with a free pitch-degree-of-freedom in the $R e$ range of $45,000 \leq R e \leq 135,000$. Springs were attached to the airfoil to simulate the effect of torsional stiffness of the structure. Limit-cycle oscillations with an amplitude around $5^{\circ}$ emerged spontaneously. The study brought to light several peculiar aspects of unsteady aerodynamics in the transitional regime. The foremost perhaps being that the standard unsteady aerodynamic model (Theodorsen 1935) fails to predict the onset of instability in this regime. The oscillations were self sustained and of very low frequency with the frequency of pitch oscillations being nearly two orders of magnitude lower than the vortex-shedding frequency. In addition, the signature of the vortex-shedding frequency was nearly absent from the pitch response. Therefore vortex-shedding was ruled out as the cause of aeroelastic oscillations. Several investigations have since followed (Poirel \& Yuan 2010; Poirel et al. 2011; Poirel \& Mendes 2014; Barnes \& Visbal 2016, 2018) and the emerging consensus points to the dynamic role of played 
by laminar separation in the unsteady dynamics. Poirel \& Yuan (2010) have referred to the phenomenon as laminar separation flutter. Yet, the questions on the cause of onset, frequency selection criterion and prediction of saturated amplitude have remained unanswered.

In the current work we attempt to answer the first two of those questions through the derived linear FSI equations. The stability analysis is carried out for the pitch degree of freedom using the structural parameters given in Poirel et al. (2008) and at several different Reynolds numbers. The mean flow is deemed to be more relevant for the stability analysis and therefore it is used as the base flow for the stability calculations. The low frequency eigenspectra of the coupled system is shown in figure 3.5 for the spring stiffness of $K_{s}=0.30$. Two sets of modes are found for the coupled FSI system marked as mode $I$ and mode $I I$. Mode $I$ is stable and exists as a complex-conjugate pair at $\operatorname{Re}=40,000$. However, it coalesces into a single zero frequency mode at higher $R e$ and crosses the stability boundary somewhere between 50,000 $<R e<60,000$. This is in good agreement with the results of the experimental campaign of Poirel et al. (2008) who found the stability boundary at $R e \approx 55,000$ for $K_{s}=0.30$. Similarly good agreement is found for the different structural parameters as well. The results of the stability analysis are peculiar since they predict only the onset of aeroelastic oscillations but not the frequency. The instability found is a divergence type instability which has a zero frequency. Thus the linear mechanism does not impose a frequency selection criterion. It is speculated that the frequency selection is provided by mode II of the aeroelastic system which may be non-linearly excited. The frequency of this mode is found to be close to the saturated LCO frequency observed in the experiments. The eigenmode for the unstable zero frequency is shown in figure 3.6. Further details of the investigation can be found in Paper 3.

\subsection{Forced response of pitching wings}

\subsubsection{Pitching laminar airfoil}

In Paper 4 the unsteady response of an NLF airfoil that is subject to small-amplitude forced pitch oscillations is investigated. The motivation for the study traces its roots to the unsteady experiments of Mai \& Hebler (2011) and Hebler et al. (2013). Again, these studies constitute possibly the first systematic studies on the aeroelasticity of laminar airfoils. The pioneering investigations showed that laminar airfoils, when subject to small-amplitude forced pitch-oscillations exhibit a non-linear response in the aerodynamic forces. This is contrary to the decades old unsteady aerodynamic theories of Theodorsen (1935) etc. who had only considered linear responses. While the focus of these early investigations had been largely in the transonic regime, their work inspired the investigations of Lokatt (2017) who performed similar investigation for laminar airfoils in subsonic flows and found strongly nonlinear aerodynamic force responses. All of the experimental investigations pointed to the variation of transition location during the unsteady experiments as the root cause of the non-linear force responses. Thus unsteady boundary-layer dynamics play an important role in aerodynamic response of NLF airfoils. 


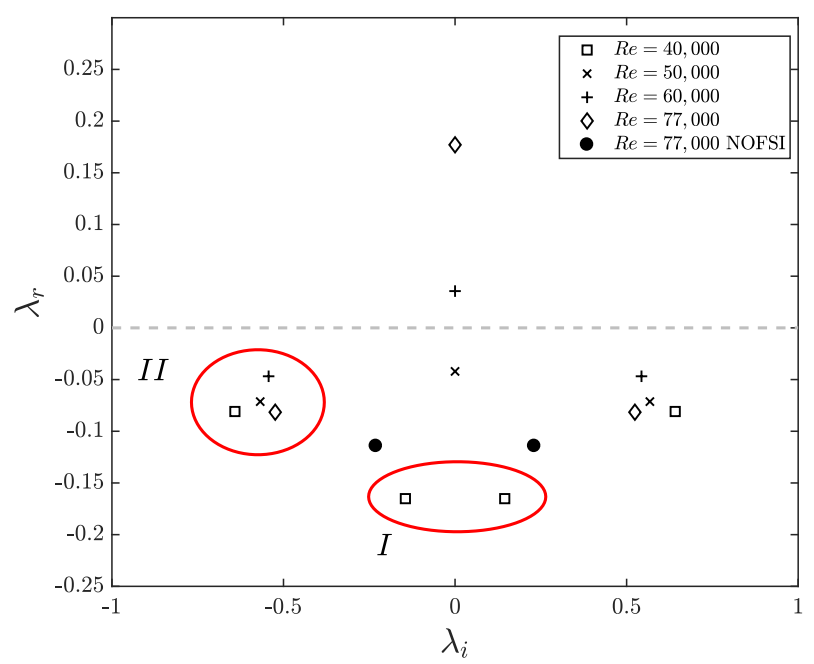

Figure 3.5: The variation of the aeroelastic modes of the coupled FSI system for different Reynolds numbers for the rotational spring stiffness constant of $K_{s}=0.30$. Also plotted in the figure is the low frequency spectrum for a stationary wing at $\operatorname{Re}=77,000$.

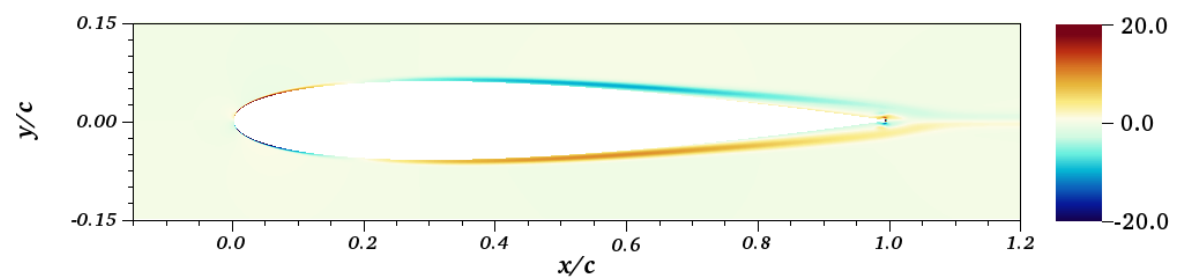

Figure 3.6: Streamwise velocity components of the eigenvector corresponding to the zero frequency unstable mode for the coupled FSI problem at $\operatorname{Re}=77,000$.

The present work investigates the unsteady boundary layers with a particular focus on unsteady transition with the aim to shed light on the phenomenon of non-linear unsteady aerodynamic response. The NLF airfoil used in the unsteady experiments (Lokatt \& Eller 2017; Lokatt 2017) has been used for the current numerical simulations. The results of the steady and unsteady experiments using this airfoil have been made available to us by Dr. Eller and Dr. Lokatt. The design of the numerical simulations was based on a study of the experimental data and prediction from an integral boundarylayer code XFOIL (Drela 1989) so as to predict the region which exhibits aerodynamic non-linearity. The chord-based Reynolds number of the simulation was set to $R e_{c}=$ $7.5 \times 10^{5}$, which is quite high for a high-fidelity unsteady simulation. Since a fully resolved DNS was computationally infeasible, a wall-resolved LES approach was used 
which resulted in a mesh size of approximately 1.4 billion points. Stationary wing simulations at different angles of attack were performed to ensure that large differences in boundary-layer transition were numerically obtained for the stationary wing cases (figure 3.7).
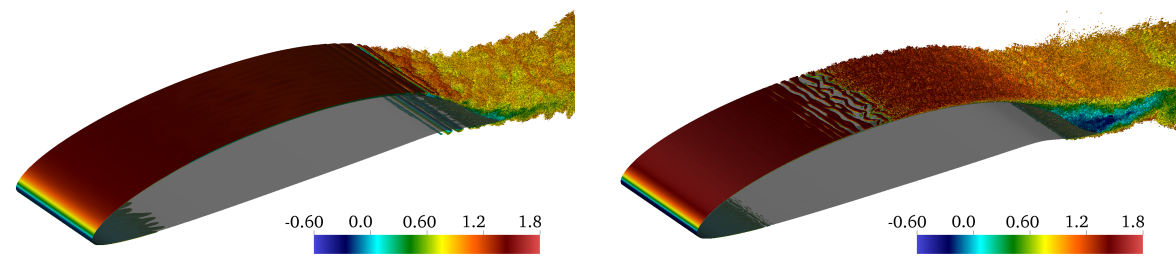

Figure 3.7: Instantaneous vortical structures identified by the $\lambda_{2}$ criterion (Jeong \& Hussain 1995) for (left) $\alpha=2.4^{\circ}$ and (right) $\alpha=4.4^{\circ}$ at $R e_{c}=750,000$.

The reduced frequency of $k=0.4$ was used for the unsteady simulations, with the reduced frequency defined as $k=\Omega b / U_{\infty}$, where $\Omega$ is the angular frequency of oscillation, $b$ is the semi-chord length and $U_{\infty}$ is the freestream velocity. A sinusoidal profile was prescribed to the angle of attack $\alpha$, with a mean of $\alpha_{0}=3.4^{\circ}$ and oscillation amplitude of $1^{\circ}$. The same $1^{\circ}$ oscillation amplitude has been used in the experimental investigations of Lokatt (2017) for several different parameters. Figure 3.8 shows the space-time plot of the unsteady, spanwise averaged wall-shear stress obtained. The time is normalized by the oscillation time period $T_{\text {osc }}$. Black contours indicate regions of zero shear stress (flow separation) and the red line is the empirically determined transition location. Clearly evident in the plot are the large changes in transition as well as the accompanying changes in flow separation. The phase portrait of transition variation with respect to $\alpha$ generates a closed loop hysteresis behavior. However, when plotted with respect to an effective angle of attack $\left(\alpha_{e}\right)$ which has a constant phase lag with respect to the instantaneous angle of attack, the hysteresis curve nearly collapses onto a single line. This collapsed curve corresponds well with the transition variation found for the stationary wing from XFOIL. The implication there being that the dynamics are nearly quasi-steady and the unsteady transition follows the steady transition curve in a phase-lagged manner. The phase portraits with respect to $\alpha$ and $\alpha_{e}$ are shown in figure 3.9. Also shown in the plot with red circles are the transition points obtained from the stationary simulations for three different $\alpha$. The collapse is not perfect, however the phase-lag clearly captures the dominant unsteady effect.

Based on the insight, a simple empirical model for the unsteady aerodynamic forces is developed where the forces may be represented as:

$$
\begin{aligned}
C_{l}(t) & =A \cos (\Omega t+\theta)+C_{l}^{\text {static }}\left(\alpha_{e}\right) . \\
\alpha(t) & =\alpha_{0}+\Delta \alpha \sin (\Omega t) \\
\alpha_{e}(t) & =\alpha_{0}+\Delta \alpha \sin (\Omega t+\phi)
\end{aligned}
$$

The model is built on the idea that the boundary-layer dynamics are quasi-steady. Therefore for a given sinusoidal variation of $\alpha$, a contribution of the the instantaneous 


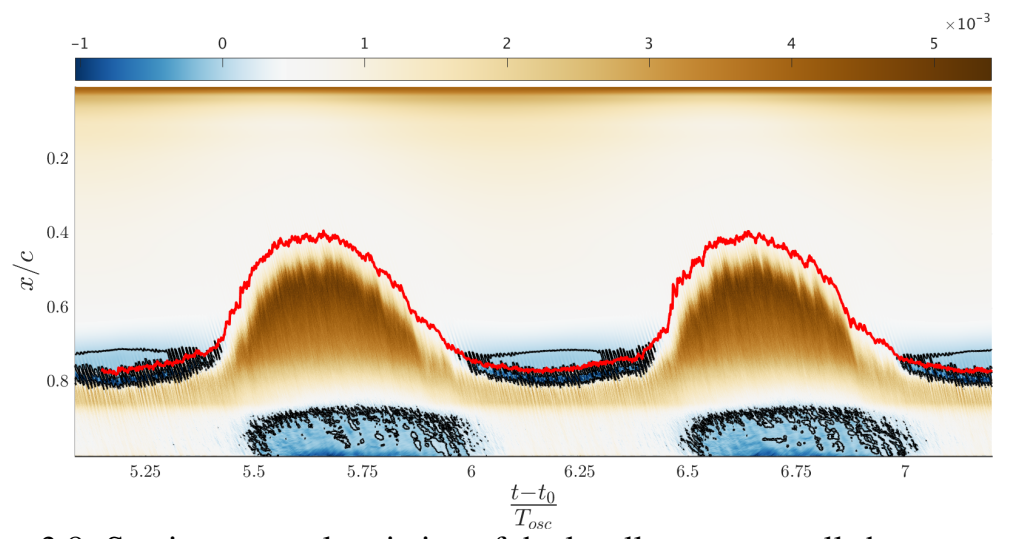

Figure 3.8: Spatio-temporal variation of the locally tangent wall shear-stress $\left(\tau_{w}\right)$. The $y$-axis represents the chord-wise location while the $x$-axis represents the normalized simulation time. The colors represent the instantaneous, spanwise-averaged wall-shear stress value, $\tau_{w}(x, t)$. Black contours denote the points of zero tangential shear stress. Empirically determined transition location (red curve) is superposed on the space-time plot.
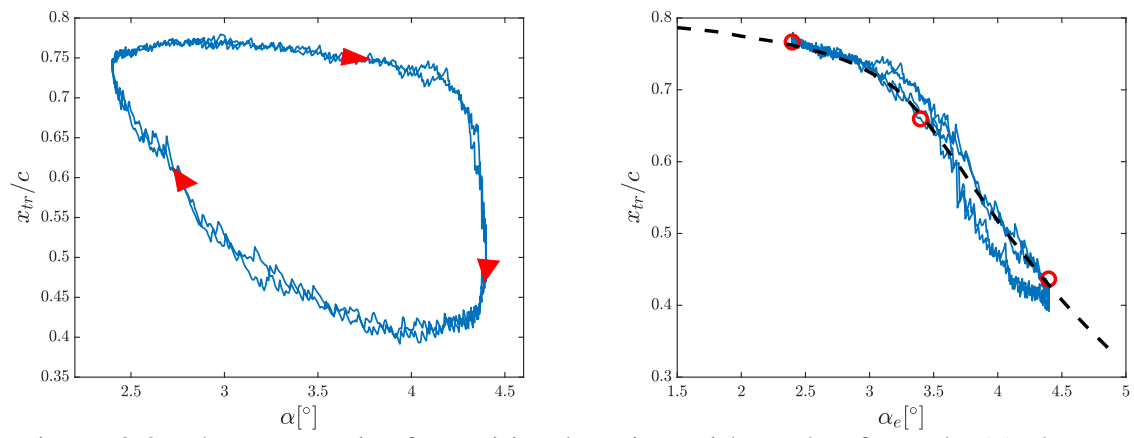

Figure 3.9: Phase-portrait of transition location with angle of attack. (a) shows the variation of transition with the instantaneous $\alpha$. Arrows in (a) indicate the forward direction in time. (b) shows transition variation with respect to an effective angle of attack $\alpha_{e}$, which is phase-lagged with respect to the instantaneous angle of attack. The blue lines represent the evaluated transition location and the black dashed line represents static transition location values obtained from XFOIL. Red circles in (b) represent the transition location obtained for simulations of a stationary wing at different $\alpha$.

unsteady aerodynamic forces must be given by the phase-lagged static aerodynamic forces. All other unsteady effects are assumed to be linear. The model contains three free parameters in the form of amplitude $A$, and phase of linear unsteady effects $\theta$, and 

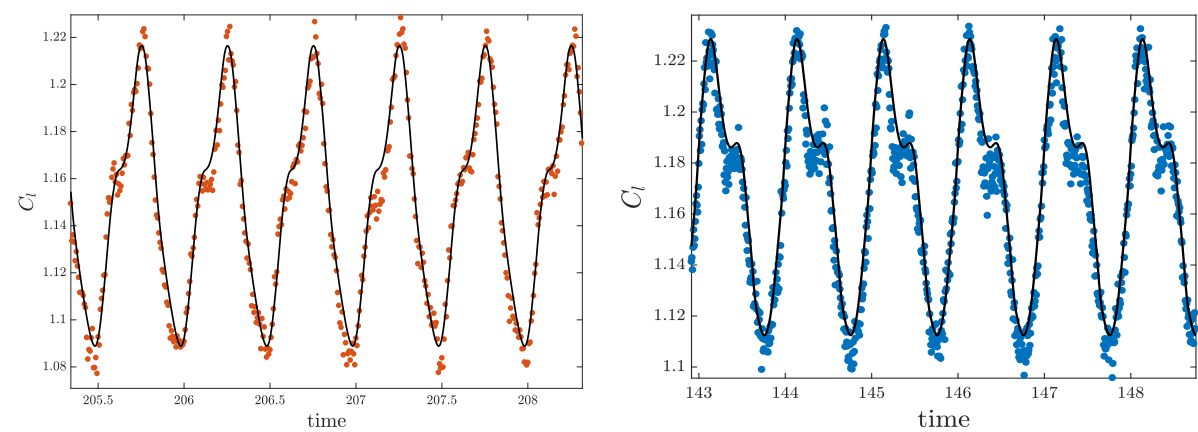

Figure 3.10: Least-squares fit of the empirical model to the experimental data. (Left) $R e_{c}=1.0 \times 10^{6}, \alpha_{0}=2.8^{\circ}$ and $k=0.1$. (Right) $\operatorname{Re}_{c}=7.5 \times 10^{5}, \alpha_{0}=3.1^{\circ}$ and $k=0.06$

the phase of the steady contribution $\phi . \alpha_{0}$ is the mean angle of attack of the oscillation. $C_{l}^{\text {static }}$ is empirically determined since inviscid theory is found to be no longer applicable in the regime of investigations. The model was used to fit the unsteady aerodynamic data from Lokatt (2017). $C_{l}^{\text {static }}$ was also obtained from the experimental data set. Figure 3.10 shows the comparison between the model and the data for two different cases. The agreement between the model and the data is found to be remarkably good.

Jon von Neumann famously remarked "with four parameters I can fit an elephant, and with five I can make him wiggle his trunk" (Dyson 2004). The current model has three free parameters so it is safely below the elephant fitting criterion of von Neumann. However, since they are essentially free parameters the model is not predictive. Nonetheless, the model isolates an important physical aspect of the unsteady dynamics i.e., the source of non-linearity. The $C_{l}^{\text {static }}$ term is the only source of non-linearity in the model and the good agreement between model and experimental data suggests that the non-linear effects have been well captured. Thus the non-linearity in the unsteady aerodynamic forces arises predominantly due to the quasi-steady term associated with the phase-lagged static aerodynamic characteristics of the wing.

\subsubsection{Forced oscillations at low Re}

Paper 5 investigates the same airfoil at a much lower $R e=100,000$. The static characteristics of the airfoil obtained from XFOIL indicate that large changes in transition occur for the airfoil even at these low transitional Reynolds numbers. However, in this regime a laminar separation bubble (LSB) develops close to the leading edge of the airfoil during the unsteady motion, which plays a dominating role in the unsteady dynamics. A space-time plot of the wall-shear stress is shown in figure 3.11, which illustrates the dynamically rich behavior of the boundary layer at these low transitional Reynolds numbers. Black regions in figure $3.11 \mathrm{~b}$ indicate negative shear stress and thus separation. Leading-edge LSB periodically forms and disappears during the oscillation cycles. The upstream and downstream motion of transition is significantly more asymmetric for this case as compared to the space-time plot in figure 3.8 where 
the asymmetry appears to be only marginal. Perhaps the most peculiar aspect of the unsteady dynamics in this flow case is the behavior of transition, which is best captured in figure 3.12 where the instantaneous visualization of the $\lambda_{2}$ structures (Jeong \& Hussain 1995) in the flow at two instances with a small time difference between them is shown. While in the figure on the left a single point of transition may be identified followed by turbulent region downstream, the figure on the right contains two distinct regions of transition separated by laminar flow between them. Thus the transition point makes a discontinuous jump at a certain time instance and two different points of transition arise in the flow.

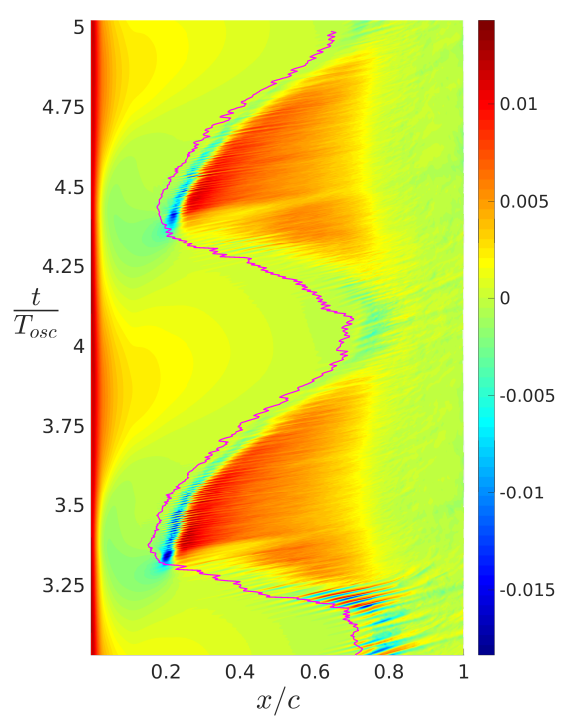

(a) Wall-shear stress $\left(\tau_{w}\right)$.

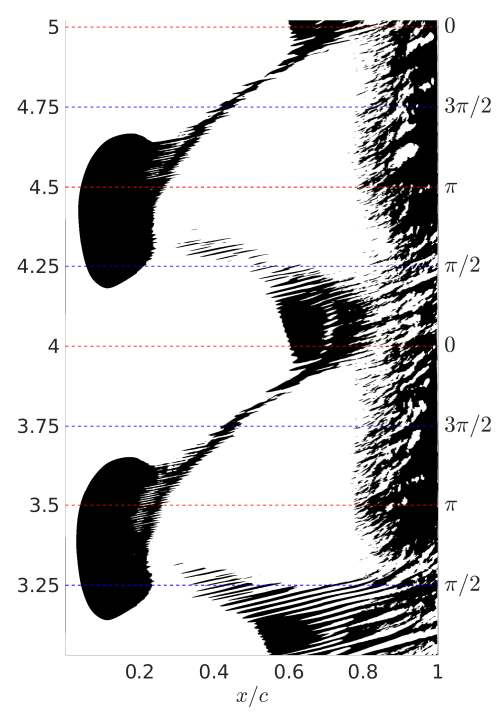

(b) Separated regions (black).

Figure 3.11: (a) Space-time plot for the wall-shear values $\left(\tau_{w}\right)$ and (b) separated flow regions. Magenta line in (a) denotes the calculated transition location. Horizontal blue dashed lines in (b) represent the extrema of the angle of attack, while the red dashed lines represent phases corresponding to mean angle of attack.

The abrupt change in transition has been investigated by analyzing the changes in the stability characteristics of the leading-edge LSB, which reaches nearly $40 \%$ reverse flow velocities. The instabilities of spatially homogeneous linear systems may be classified as convective or absolute instabilities. In brief, convective instabilities occur when small disturbances grow while traveling downstream from their point of origin. On the other hand, absolute instabilities are characterized by the growth of disturbance at their point of origin. The instabilities are often associated with the concept of group velocities of perturbations. While convective instabilities typically 

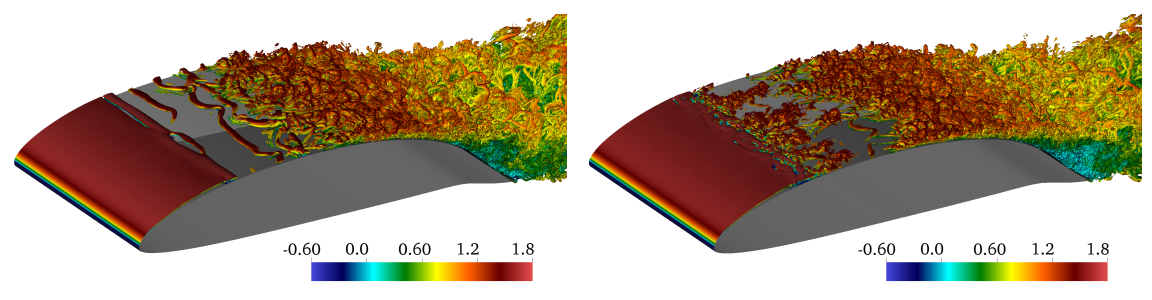

Figure 3.12: Comparison of boundary-layer transition at two different time instants of $t / T_{o s c}=3.33$ (left) and $t / T_{o s c}=3.35$ (right) as visualized through the $\lambda_{2}$ structures. The color represents streamwise velocity.

have a positive group velocity and hence travel away from their point of origin as they amplify, absolute instabilities exhibit a zero group velocity and therefore do not travel downstream as they amplify. For a more detailed exposition, the reader is referred to Briggs (1964) where the original classification is made and to a more recent reference Bers (2016). A local stability analysis with the Orr-Sommerfeld equations can be performed to investigate the convective and absolute character of the boundary layer. The local stability analysis in the reverse flow region indicated that initially the LSB is convectively unstable but during the oscillation a region of absolute instability develops inside the LSB. Therefore the LSB changes character from being convectively unstable to absolutely unstable. It is conjectured that this change in character causes a competition between the two different instability mechanisms. The convective instability amplifies as it travels downstream and causes transition once the disturbances reach a high enough amplitude. Initially when the flow is absolutely stable, only the convective instability causes transition. As the LSB grows the flow is both convectively and absolutely unstable. Eventually disturbances in the reverse flow regions grow large enough without being convected downstream and cause transition over the separated shear layer. At this instance, two points of transition arise - one due to the absolute instability and one due to the convective instability. Figure 3.13 shows the variation of the absolute growth rate with the streamwise coordinate found at time $t / T_{\text {osc }}=3.32$. A pocket of absolute instability is found in the rear of the LSB. This marginally precedes the time stamps shown in figure 3.12 .

The existence of an absolute instability is identified by the location of the saddle point of $\omega$ in the complex $k$ plane, where $\omega$ is the complex angular frequency associated with the complex wavenumber $k$ of the disturbance. However, for spatially inhomogeneous systems, Huerre \& Monkewitz (1990) and Chomaz et al. (1991) have shown that an additional criterion arises, which requires that the unstable angular frequency $\omega$ simultaneously exhibits a saddle in the complex $X$ plane, where $X$ is a complex streamwise coordinate. The frequency at which these two saddles occur simultaneously gives the global absolute frequency $\omega_{g}$ of the spatially inhomogeneous system. Such an approximation requires solutions in a complex space, which are typically not available directly through simulation data. However, as done by Cooper \& Crighton (2000) and Juniper et al. (2011), the variation of the absolute frequency in the complex $X$ 

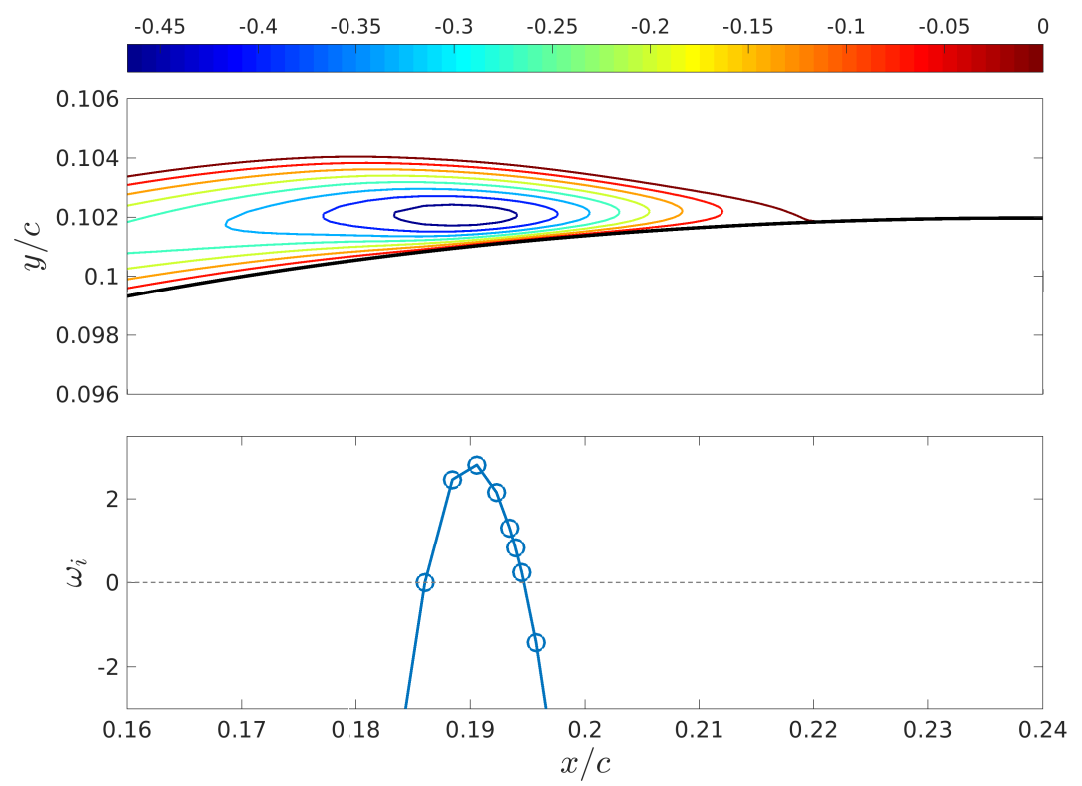

Figure 3.13: Top: Contours of negative stream-wise velocity near the leading-edge of the airfoil at $t / T_{\text {osc }}=3.32$. Bottom: Variation of absolute growth rate with the chord-wise location.

plane may be determined from information on the real $X$ axis and then assuming an analytic continuation of the absolute frequency. In Cooper \& Crighton (2000); Juniper et al. (2011), the authors fit the spatial variation of the complex absolute frequency $\omega_{0}$ obtained for real $X$ with a Padé approximant (rational polynomials). The saddle in complex $X$ is trivially evaluated once the analytical Padé approximant is known. The same procedure is followed in the current work. The Padé approximants of the absolute frequencies is shown in figure 3.14 and the associated saddle is shown in figure 3.15. The saddle in the complex $X$ plane is found to lie very close to the real axis with the global frequency found to be $\omega_{g}=17.83+2.741 i$. 

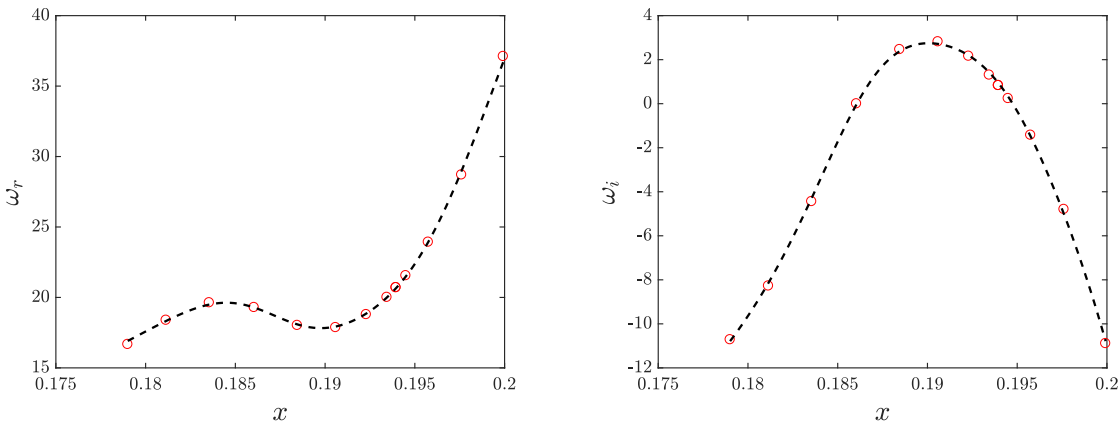

Figure 3.14: (Left) Absolute angular frequency (real part) and (right) absolute growth rate (imaginary part) along with the best fit Padé approximation (dashed lines).
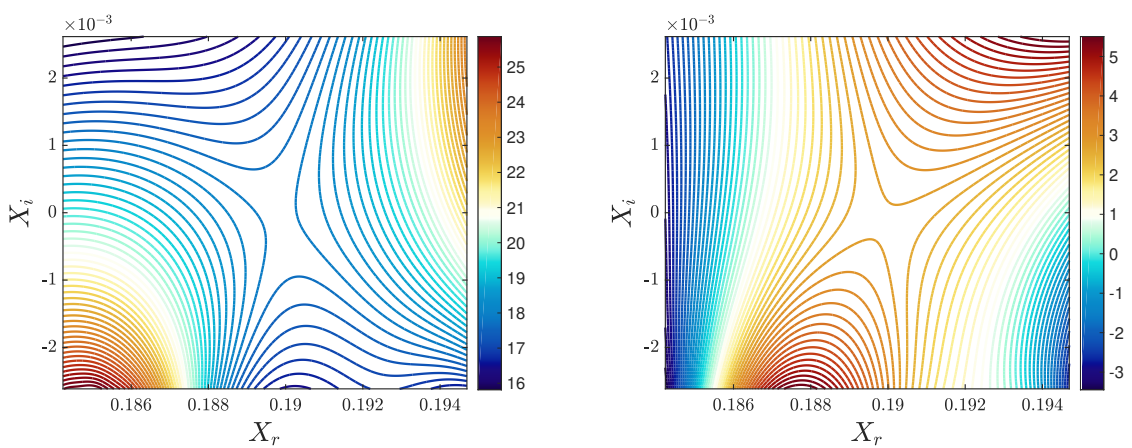

Figure 3.15: Saddle in the complex $X$ plane for (left) absolute angular frequency (real part) and (right) absolute growth rate (imaginary part). 
ChAPTER 4

\section{Conclusions and outlook}

\subsection{Conclusions}

The "evolve and filter" technique for the stabilization of spectral-element methods is analyzed and it is found that the filter operation causes an undesirable loss of divergencefree quality of the solution. This loss of divergence is shown to be particularly large for the test case of a double shear layer. An alternate formulation of the stabilization called the relaxation-term (RT) stabilization is shown to overcome the drawbacks of the explicit filtering technique, while maintaining the simplicity of the original explicit filter. This RT stabilization technique is very closely related to explicit filtering, with the two operations being equivalent to the leading order in time. Stability limits of the RT stabilization are explored and is shown to be stable within the practical parameter range.

The linear stability equations for a rigid-body motion FSI problem are derived and validated. Linear stability analysis is then used to investigate the coupled FSI problem of a rotating cylinder with an attached splitter-plate. The system undergoes a symmetry breaking bifurcation in which the cylinder rotates and the splitter-plate equilibrates at a non-zero angle to the flow. The linear analysis showed that the onset of the symmetry breaking occurs due to a zero frequency linearly unstable mode which causes a continuous rotation of the system till the new equilibrium is reached. The linear equations are also used to investigate the structural sensitivity of an oscillating cylinder which is found to change significantly as compared to a stationary cylinder, when the natural frequency of the spring-mass system is close to the unstable frequency of the coupled system.

The linear FSI equations are then used to investigate the onset of pitch-oscillations for a NACA0012 airfoil at transitional Reynolds numbers. The linear instability analysis predicts an onset of aeroelastic instability which is in close correspondence with the experimental observations of Poirel et al. (2008). However, the instability predicted by the linear analysis is a divergence type instability with zero frequency. Non-linear results with a smaller domain corroborate the results of the linear analysis and exponential growth of a zero frequency mode is also found in the non-linear simulations. The linear results therefore do not provide a frequency selection criteria and it is conjectured that a stable low frequency aeroelastic mode is non-linearly triggered which may provide the frequency selection criteria. 
One-way coupled systems are investigated for the case of small-amplitude forced pitch-oscillations of an airfoil at two different Reynolds numbers. In the first case the investigation is set in the context of NLF airfoils for which the aerodynamic responses have recently been found to disagree with the classical linear unsteady aerodynamic theory. Simulations performed at $R e=7.5 \times 10^{5}$ showed a large variation of transition location throughout the pitch cycle. This variation however was found to exhibit a phase-lagged quasi-steady behavior which corresponded well with the variation of transition with angle of attack for a stationary airfoil. Based on the insight an empirical model is developed for the unsteady aerodynamic forces which matches well with the unsteady experimental data. The model isolates the source of non-linearity entirely to the quasi-steady term which is associated with the static characteristics of the airfoil.

In the second case, the same airfoil is investigated at much lower Reynolds number of $R e=1.0 \times 10^{5}$. The boundary layer is found to exhibit a dynamically rich behavior with large variations in transition and separation. The transition is found to change abruptly during the unsteady motion. The formation of a leading-edge laminar separation bubble (LSB) dominates the unsteady dynamics and it is conjectured that a change in the stability characteristics of the leading-edge LSB from convective to absolute instability is the cause of the abrupt change in transition during the oscillation cycle.

Finally, the flow over a stationary NACA 4412 airfoil is studied at different Reynolds numbers with the aim of better understanding the boundary-layer evolution in non-equilibrium pressure gradient boundary layers. Flow cases at a chord-based Reynolds number varying from $R e_{c}=100,000$ to $R e_{c}=1,000,000$ are compared at different streamwise locations. It is found that the effect of the streamwise pressure gradients is higher at low Reynolds numbers, leading to greater energy in the larger structures present in the outer part of the turbulent boundary layer.

\subsection{Outlook}

The current work started with some open questions in unsteady aerodynamics, with the focus of the studies being primarily limited to the regime where the classical aeroelastic models are known to fail. While some of the work has provided insight, if not complete answers, it also raises several open questions to be answered in the future.

Perhaps foremost is the extension of the current linear FSI equations to general structural motions. While the derivation of the linearized FSI equations from the perspective of the fluid variables was performed without assuming any particular form of the interface deformation, its validation is limited to rigid-body motions. A validation for a general case is highly warranted. In particular the vanishing of the "added-stiffness" terms arising in the formulation of Fanion et al. (2000) needs to be numerically confirmed in a general case. This is an important step forward since the application of the Taylor expansion based linearization appears to have been held back due to the "added-stiffness" terms which require evaluation of higher order derivatives on the base flow state. A validation of their vanishing nature would lend the method to more widespread use.

While the adjoint analysis of Paper $\mathbf{2}$ provided a structural map of the eigenvalue sensitivity, no effort was made toward the control of the oscillating cylinder by making 
use of the adjoint information. This presents a natural next step of the investigation. However, it fell outside the scope of the current thesis work.

Aeroelastic instability analysis at transitional Reynolds numbers provides several different directions for the extension of the work. Suppression of aeroelastic instability due to high freestream turbulence has been reported in the literature (Poirel et al. 2008; Yuan et al. 2015) and it would be valuable to know if this effect is also captured by the linear stability analysis. The adjoint based analysis may possibly be used in this case as well to efficiently suppress limit-cycle oscillations. Recently Barnes \& Visbal (2018a) have used plasma actuators to suppress laminar separation flutter. An adjoint based analysis may possibly provide the most efficient way of suppression.

For the case of unsteady laminar airfoils, while the current work provides insight into the source of non-linear behavior through the phase-lagged boundary-layer dynamics, the question of predicting the phase lag has been left completely unanswered. Phase-lag is a very recurring idea in unsteady aerodynamics as suggested by the works of Ericsson \& Reding (1986, 1988a); Goman \& Khrabrov (1994); Barnes \& Visbal (2018) etc. However, prediction of the phase lag outside the linear unsteady aerodynamic regime does not appear to be addressed in the literature. Since the variation of transition location in the unsteady scenario has been shown to correspond well with the static characteristics of the airfoil, might the information of the phase lag also be available from its static characteristics? At the moment it does not seem to be entirely clear if it would.

The low Reynolds number forced oscillation case presents dynamically rich behavior and the interaction of several different physical aspects - transition motion, LSB, stability changes, and trailing-edge separation to name a few. Quite possibly several of these effects may be specific to the particular flow case. However, one particular aspect stands out which has not been investigated in the current work, and that is the asymmetry of the upstream and downstream motion of transition. In the higher $R e$ case this asymmetry was only marginal, but was very pronounced for the low $R e$ case. The criteria for the selection of velocity of the turbulent front may have implications for the prediction of unsteady dynamics in other flow cases as well, and is well worthy of an investigation. 


\section{Acknowledgements}

This thesis marks the completion of the PhD chapter of my life, which has unfolded over the last five years. I was fortunate to have had three advisors, Philipp Schlatter, Ardeshir Hanifi and Dan Henningson, to guide me through it, and have benefited greatly from their knowledge and experience. My foremost gratitude goes to my main advisor Dan, for accepting me into his research group. Not only has he given me the opportunity and freedom to develop my ideas and grow as a researcher, he has always been enthusiastic about my progress and the next direction of research. He has been supportive when things have looked bleak, while still pushing me forward. His confidence is contagious, and has often pushed me towards things I did not know I was capable of. He will forever be a source of inspiration.

Ardeshir has always had an open door for me, and I could always walk into his office without a second thought and ask the most ridiculous of questions. I was always heard with patience and my ideas and questions never dismissed. My earliest scientific discussions happened with Philipp, and my ignorance in numerics must have been painfully obvious. Yet, my ideas and suggestions were never disparaged. I am grateful to them both for allowing room for my ignorance and letting me grow. It was one of the greatest luxuries that could be afforded to a doctoral student. Adam Peplinski has been my guide for Nek5000, MPI and coding in general. I appreciate all the help that he has provided and can not imagine figuring out the code without him. I would also like to thank Paul Fischer. My interactions with him have been few and far in between, but he has provided help with the code in a few very crucial moments during the $\mathrm{PhD}$. I would also like to acknowledge the members of the NFFP project, Dr. Roger Larsson, Dr. David Eller and Dr. Mikaela Lokatt for all the interesting discussions on aeroelasticity. Some of Mikaela's inputs have played a decisive role determining the direction of the current research.

A thanks goes out to Mattias, Elektra and Nicolas for proofreading this thesis. My carelessness with writing would have been a lot more obvious without their corrections.

A heartfelt thanks goes out to all the new friends I made during this journey. It has been an incredible time with some really great friendships. There have been some that were momentary and some, that I hope will last forever. Thank you all for being there in this part of my life. You have all made things a bit more colorful, and this story may have unfolded very differently without you. 
A special mention goes out to my friends and cousins back home, who I always meet during my vacations, and the few in New York, who I met on my trips to the US. It was always a pleasure to see you all.

Perhaps most importantly, my deepest gratitude goes to my parents and my sister, for their patience, unconditional love and support throughout my life. It has been a wandering journey so far and I have always found them standing by me, through thick and thin.

Finally, I would like to acknowledge the financial support provided for this work by Vinnova through the NFFP project UMTAPS, with grant number 2014-00933, the European Research Council under grant agreement 694452-TRANSEP-ERC-2015AdG and the Swedish e-Science Research Centre (SeRC). The computations were performed on resources provided by the Swedish National Infrastructure for Computing (SNIC) at the PDC Center for High Performance Computing at the Royal Institute of Technology (KTH). Simulations have also been performed at the High Performance Computing Center, Stuttgart (HLRS), with the computer time provided by the $15^{\text {th }}$ PRACE Project Access Call (number 2016163965). 


\section{Bibliography}

Anderson, J. D. 1989 Introduction To Flight. United States: McGraw-Hill Inc.

Bagheri, S., Mazzino, A. \& Bottaro, A. 2012 Spontaneous symmetry breaking of a hinged flapping filament generates lift. Physical Review Letters 109 (15), 154502.

BARNES, C. J. \& Visbal, M. 2016 High-fidelity LES simulations of self-sustained pitching oscillations on a NACA0012 airfoil at transitional Reynolds numbers. In 54th AIAA Aerospace Sciences Meeting.

Barnes, C. J. \& VisbaL, M. R. $2018 a$ Mitigation of laminar separation flutter using plasma-based actuators. In 2018 Flow Control Conference.

BARNes, C. J. \& Visbal, M. R. $2018 b$ On the role of flow transition in laminar separation flutter. Journal of Fluids and Structures 77, 213 - 230.

Benjamin, T. B. 1959 Shearing flow over a wavy boundary. Journal of Fluid Mechanics 6 (2), 161-205.

Benjamin, T. B. 1960 Effects of a flexible boundary on hydrodynamic stability. Journal of Fluid Mechanics 9 (4), 513-532.

BERs, A. 2016 Plasma physics and fusion plasma electrodynamics. Oxford University Press.

Bisplingoff, L. R., Ashley, H. \& Halfman, R. L. 1983 Aeroelasticity. Dover Publications INC.

BlakesLeE, S. 1986 Voyager succeeds in historic flight. The New York Times .

Bobke, A., Vinuesa, R., ÖrLü, R. \& Schlatter, P. 2017 History effects and near equilibrium in adverse-pressure-gradient turbulent boundary layers. Journal of Fluid Mechanics $\mathbf{8 2 0}$, 667-692.

Brandt, L., Schlatter, P. \& Henningson, D. S. 2004 Transition in boundary layers subject to free-stream turbulence. Journal of Fluid Mechanics 517.

Briggs, R. J. 1964 Electron-Stream Interaction with Plasmas. The MIT Press, Cambridge, Massachusetts.

Bushnell, D. M. 2003 Aircraft drag reduction - a review. Proceedings of the Institution of Mechanical Engineers, Part G: Journal of Aerospace Engineering 217 (1), 1-18.

Cano-Lozano, J. C., Tchoufag, J., Magnaudet, J. \& Martínez-Bazán, C. 2016 A global stability approach to wake and path instabilities of nearly oblate spheroidal rising bubbles. Physics of Fluids 28 (1), 014102.

Carr, L. W., McAlister, K. W. \& McCroskey, W. J. 1977 Analysis of the development of dynamic stall based on oscillating airfoil experiments. Tech. Rep.. NASA Ames Research Center; Moffett Field, CA, United States.

Causin, P., Gerbeau, J. \& Nobile, F. 2005 Added-mass effect in the design of partitioned algorithms for fluid-structure problems. Computer Methods in Applied Mechanics and Engineering 194 (42), 4506 - 4527. 
Cayley, S. G. 1809-10 On aerial navigation. http://ota.ox.ac.uk/text/3075.html.

Chin, C., NG, H., Blackburn, H., Monty, J. \& Ooi, A. 2015 Turbulent pipe flow at $\operatorname{R}_{\tau}=1000$ : A comparison of wall-resolved large-eddy simulation, direct numerical simulation and hot-wire experiment. Computers and Fluids 122, 26 - 33.

Chomaz, J.-M., Huerre, P. \& Redekopp, L. G. 1991 A frequency selection criterion in spatially developing flows. Studies in Applied Mathematics 84 (2), 119-144.

Clauser, F. H. 1954 Turbulent boundary layers in adverse pressure gradients. Journal of Aeronautical Sciences 21 (2), 91-108.

Cooper, A. J. \& Crighton, D. G. 2000 Global modes and superdirective acoustic radiation in low-speed axisymmetric jets. European Journal of Mechanics - B/Fluids 19 (5), 559 - 574.

Coorke, T. C. \& Thомas, F. O. 2015 Dynamic stall in pitching airfoils: Aerodynamic damping and compressibility effects. Annual Review of Fluid Mechanics 47 (1), 479-505.

Cossu, C. \& Morino, L. 2000 On the instability of a spring-mounted circular cylinder in a viscous flow at low reynolds numbers. Journal of Fluids and Structures 14 (2), 183 - 196

Crisler, W. C., Krothapali, A. \& Lourenco, L. M. 1994 PIV investigation of high speed flow over a pitching airfoil. In 32nd Aerospace Sciences Meeting and Exhibit, Aerospace Sciences Meetings, AIAA. Renvo, N.V., USA.

Deville, M. O., Fischer, P. F. \& Mund, E. H. 2002 High-Order Methods for Incompressible Fluid Flow. Cambridge Monographs on Applied and Computational Mathematics 9. Cambridge University Press.

Dowell, E. H. \& Hall, K. C. 2001 Modeling of fluid-structure interaction. Annual Review of Fluid Mechanics 33 (1), 445-490.

Drela, M. 1989 XFOIL: An Analysis and Design System for Low Reynolds Number Airfoils, pp. 1-12. Berlin, Heidelberg: Springer Berlin Heidelberg.

Dunne, R. \& McKeon, B. J. 2015 Dynamic stall on a pitching and surging airfoil. Experiments in Fluids 56 (8), 157.

Dyson, F. 2004 A meeting with Enrico Fermi. Nature 427 (6972), 297.

Eitel-Amor, G., Örlü R. \& Schlatter, P. 2014 Simulation and validation of a spatially evolving turbulent boundary layer up to $\operatorname{Re}_{\theta}=8300$. International Journal of Heat and Fluid Flow 47, 57-69.

El Khoury, G. K., Schlatter, P., Noorani, A., Fischer, P. F., Brethouwer, G. \& Johansson, A. V. 2013 Direct numerical simulation of turbulent pipe flow at moderately high Reynolds numbers. Flow, Turbulence and Combustion 91 (3), 475-495.

Ericsson, L. \& Reding, J. 1986 Fluid dynamics of unsteady separated flow. Part i. Bodies of revolution. Progress in Aerospace Sciences 23 (1), 1 - 84.

Ericsson, L. \& Reding, J. 1987 Fluid dynamics of unsteady separated flow. Part ii. Lifting surfaces. Progress in Aerospace Sciences 24 (4), 249 - 356.

Ericsson, L. \& Reding, J. 1988a Fluid mechanics of dynamic stall part i. Unsteady flow concepts. Journal of Fluids and Structures 2 (1), 1 - 33.

Ericsson, L. \& Reding, J. $1988 b$ Fluid mechanics of dynamic stall part ii. Prediction of full scale characteristics. Journal of Fluids and Structures 2 (2), 113 - 143.

ERn, P., FrÉdÉric, R., FAbre, D. \& Magnaudet, J. 2012 Wake-induced oscillatory paths of bodies freely rising or falling in fluids. Annual Review of Fluid Mechanics 44 (1), 97-121.

Fabre, D., Assemat, P. \& Magnaudet, J. 2011 A quasi-static approach to the stability of the path of heavy bodies falling within a viscous fluid. Journal of Fluids and Structures 27 (5), 758 767, iUTAM Symposium on Bluff Body Wakes and Vortex-Induced Vibrations (BBVIV-6). 
Fanion, T., Fernández, M., A. \& Le TAllec, P. 2000 Deriving adequate formulations for fluidstructure interaction problems: from ALE to transpiration. Revue Européenne des Éléments Finis 9 (6-7), 681-708.

Fernández, M. A. \& Le TAllec, P. $2003 a$ Linear stability analysis in fluid-structure interaction with transpiration. Part i: Formulation and mathematical analysis. Computer Methods in Applied Mechanics and Engineering 192 (43), 4805 - 4835.

Fernández, M. A. \& Le Tallec, P. $2003 b$ Linear stability analysis in fluid-structure interaction with transpiration. Part ii: Numerical analysis and applications. Computer Methods in Applied Mechanics and Engineering 192 (43), 4837 - 4873.

Fischer, P., Heisey, K. \& Min, M. 2015 Scaling Limits for PDE-Based Simulation (Invited). In 22nd AIAA Computational Fluid Dynamics Conference, AIAA AVIATION Form. AIAA.

Fischer, P., Schmitт, M. \& Tomboulides, A. 2017 Recent Developments in Spectral Element Simulations of Moving-Domain Problems, pp. 213-244. New York, NY: Springer New York.

Fischer, P. F., Lottes, J. W. \& Kerkemeier, S. G. 2008 Nek5000 web page. http://nek5000. mcs.anl.gov.

Freund, J. B. 2014 Numerical simulation of flowing blood cells. Annual Review of Fluid Mechanics 46 (1), 67-95.

GARRICK, I. E. \& ReED III, W. H. 1981 Historical development of aircraft flutter. Journal of Aircraft 18 (11), 897-912.

Gianetti, F. \& Luchini, P. 2007 Structural sensitivity of the first instability of the cylinder wake. Journal of Fluid Mechanics 581, 167-197.

Gibвs-Sмiтн, C. H. 1962 Sir George Cayley: 'Father of aerial navigation' (1773-1857). Notes and Records of the Royal Society 17 (1), 36-56.

Glauert, H. 1930 The force and moment on an oscillating aerofoil. In Vorträge aus dem Gebiete der Aerodynamik und verwandter Gebiete: Aachen 1929 (ed. A. Gilles, L. Hopf \& T. v. Kármán), pp. 88-95. Berlin, Heidelberg: Springer Berlin Heidelberg.

Goman, M. \& Khrabrov, A. 1994 State-space representation of aerodynamic characteristics of an aircraft at high angles of attack. Journal of Aircraft 31 (5), 1109-1115.

Goza, A., Colonius, T. \& SAdER, J. E. 2018 Global modes and nonlinear analysis of inverted-flag flapping. Journal of Fluid Mechanics 857, 312-344.

Green, J. E. 2008 Laminar flow control-back to the future. 38th Fluid Dynamics Conference and Exhibit, Fluid Dynamics and Co-located Conferences, AIAA 3738.

HaLfman, R. L. 1952 Experimental aerodynamic derivatives of a sinusoidally oscillating airfoil in two-dimensional flow. Tech. Rep.. National Advisory Committee for Aeronautics; Washington, DC, United States.

Hebler, A., Schojda, L. \& MAI, H. 2013 Experimental investigation of the aeroelastic behavior of a laminar airfoil in transonic flow. In Proceedings IFASD.

HeIL, M. \& Hazel, A. L. 2011 Fluid-structure interaction in internal physiological flows. Annual Review of Fluid Mechanics 43 (1), 141-162.

HeinRichs, W. 1996 High-order time splitting for the Stokes equations. Journal of Scientific Computing 11 (4), 397-410.

Hirt, C., Amsden, A. \& Cook, J. 1974 An arbitrary Lagrangian-Eulerian computing method for all flow speeds. Journal of Computational Physics 14 (3), 227 - 253.

Ho, L.-W. \& Patera, A. T. 1990 A Legendre spectral element method for simulation of unsteady incompressible viscous free-surface flows. Computer Methods in Applied Mechanics and Engineering 80 (1), 355 - 366. 
Ho, L.-W. \& PATERA, A. T. 1991 Variational formulation of three-dimensional viscous free-surface flows: Natural imposition of surface tension boundary conditions. International Journal for Numerical Methods in Fluids 13 (6), 691-698.

Hosseini, S. M., Vinuesa, R., Schlatter, P., Hanifi, A. \& Henningson, D. S. 2016 Direct numerical simulation of the flow around a wing section at moderate Reynolds number International Journal of Heat and Fluid Flow 61, 117 - 128.

Huerre, P. \& Monkewitz, P. A. 1990 Local and global instabilities in spatially developing flows. Annual Review of Fluid Mechanics 22 (1), 473-537.

Jeong, J. \& Hussain, F. 1995 On the identification of a vortex. Journal of Fluid Mechanics 285.

JiMÉNEZ, J. \& HoyAs, S. 2008 Turbulent fluctuations above the buffer layer of wall-bounded flows Journal of Fluid Mechanics 611, 215-236.

Juniper, M. P., Tammisola, O. \& Lundell, F. 2011 The local and global stability of confined planar wakes at intermediate Reynolds number. Journal of Fluid Mechanics 686, 218-238.

Karman, T. V. \& SEars, W. R. 1938 Airfoil theory for non-uniform motion. Journal of Aeronautical Sciences 5 (10), 379-390.

KIM, J., Moin, P. \& Moser, R. 1987 Turbulence statistics in fully developed channel flow at low Reynolds number. Journal of Fluid Mechanics 177, 133-166.

Kleusberg, E. 2017 Wind turbine simulations using spectral elements. Licentiate thesis, Royal Institute of Technology (KTH), Stockholm, Sweden.

KüttLeR, U. \& Wall, W. A. 2008 Fixed-point fluid-structure interaction solvers with dynamic relaxation. Computational Mechanics 43 (1), 61-72.

Lācis, U., Brosse, N., Ingremeau, F., Mazzino, A., Lundell, F., Kellay, H. \& Bagheri, S. 2014 Passive appendages generate drift through symmetry breaking. Nature communications $\mathbf{5}$, 5310.

LANDAHL, M. T. 1962 On the stability of a laminar incompressible boundary layer over a flexible surface. Journal of Fluid Mechanics 13 (4), 609-632.

Lee, M. \& Moser, R. D. 2015 Direct numerical simulation of turbulent channel flow up to $R e_{\tau} \approx 5200$. Journal of Fluid Mechanics 774, 395-415.

Leishman, J. G. 2000 Principles of Helicopter Aerodynamics. Cambridge University Press.

Lокатт, M. 2017 On aerodynamic and aeroelastic modeling for aircraft design. Doctoral thesis, KTH Royal Institute of Technology.

Lokatt, M. \& Eller, D. 2017 Robust viscous-inviscid interaction scheme for application on unstructured meshes. Computers \& Fluids 145, 37 - 51.

Lombard, J.-E. W., Moxey, D., Sherwin, S. J., Hoessler, J. F. A., Dhandapani, S. \& Taylor, M. J. 2016 Implicit Large-Eddy Simulation of a Wingtip Vortex. AIAA Journal 54, 506-518.

Maday, Y. \& Patera, A. T. 1989 Spectral element methods for the incompressible Navier-Stokes equations. In State-of-the-art surveys on computational mechanics (A90-47176 21-64). New York, American Society of Mechanical Engineers, 1989, p. 71-143. Research supported by DARPA., pp. 71-143.

MaI, H. \& Hebler, A. 2011 Aeroelasticity of a laminar wing. In Proceedings IFASD. Paris.

McCroskey, W. J. 1981 Phenomenon of dynamic stall. Tech. Rep.. NASA Ames Research Center; Moffett Field, CA, United States.

McCroskey, W. J. 1982 Unsteady airfoils. Annual Review of Fluid Mechanics 14 (1), 285-311.

McCroskey, W. J., Carr, L. W. \& McAlister, K. W. 1976 Dynamic stall experiments on oscillating airfoils. AIAA Journal 14 (1), 57 - 63.

McCroskey, W. J., McAlister, K. W., Carr, L. W. \& Pucci, S. L. 1982 An experimental study 
of dynamic stall on advanced airfoil sections. Volume 1: Summary of the experiment. Tech. Rep.. NASA Ames Research Center, Moffett Field, CA, United States.

MeIJer Drees, J. 1979 One thousand years of rotating wings. In Fifth European rotorcraft and powered lift aircraft forum, p. 35. Amsterdam, The Netherlands, september $4-7^{\text {th }}$.

Mittal, R., Seshadri, V. \& Udaykumar, H. S. 2004 Flutter, tumble and vortex induced autorotation. Theoretical and Computational Fluid Dynamics 17 (3), 165-170.

MitTal, S. \& Singh, S. 2005 Vortex-induced vibrations at subcritical Re. Journal of Fluid Mechanics 534, 185-194.

Moser, R. D., Kim, J. \& Mansour, N. N. 1999 Direct numerical simulation of turbulent channel flow up to $\mathrm{R}_{\tau}=590$. Physics of Fluids 11 (4), 943-945.

Mueller, T. J. \& DeLaurier, J. D. 2003 Aerodynamics of small vehicles. Annual Review of Fluid Mechanics 35 (1), 89-111.

NASA 2014 Sr-71 blackbird. https://www. nasa.gov/centers/armstrong/news/ FactSheets/FS-030-DFRC.html.

NAVrose \& MitTaL, S. 2016 Lock-in in vortex-induced vibration. Journal of Fluid Mechanics 794, 565-594.

Patera, A. T. 1984 A spectral element method for fluid dynamics: Laminar flow in a channel expansion. Journal of Computational Physics 54 (3), 468 - 488.

Pfister, J.-L., Marquet, O. \& CARini, M. 2019 Linear stability analysis of strongly coupled fluid-structure problems with the Arbitrary-Lagrangian-Eulerian method. Computer Methods in Applied Mechanics and Engineering 355, 663 - 689.

Poirel, D., Harris, Y. \& Benaissa, A. 2008 Self-sustained aeroelastic oscillations of a NACA0012 airfoil at low-to-moderate Reynolds numbers. Journal of Fluids and Structures 24 (5), 700 $-719$.

Poirel, D. \& Mendes, F. 2014 Experimental small-amplitude self-sustained pitch-heave oscillations at transitional Reynolds numbers. AIAA Journal 52 (8), 1581-1590.

Poirel, D., Métivier, V. \& Dumas, G. 2011 Computational aeroelastic simulations of selfsustained pitch oscillations of a NACA0012 at transitional Reynolds numbers. Journal of Fluids and Structures 27 (8), 1262 - 1277.

Poirel, D. \& YuAN, W. 2010 Aerodynamics of laminar separation flutter at a transitional Reynolds number. Journal of Fluids and Structures 26 (7), 1174 - 1194.

Prasanth, T. K. \& Mittal, S. 2008 Vortex-induced vibrations of a circular cylinder at low Reynolds numbers. Journal of Fluid Mechanics 594, 463-491.

RAINEY, A. G. 1957 Measurement of aerodynamic forces for various mean angles of attack on an airfoil oscillating in pitch and on two finite-span wings oscillating in bending with emphasis on damping in the stall. Tech. Rep.. National Advisory Committee for Aeronautics. Langley Aeronautical Lab.; Langley Field, VA, United States.

Riley, J. J., Gad-el HaK, M. \& Metcalfe, R. W. 1988 Complaint coatings. Annual Review of Fluid Mechanics 20 (1), 393-420.

Schlatter, P. 2001 Direct numerical simulation of laminar-turbulent transition in boundary layer subject to free-stream turbulence. Diploma thesis, Royal Institute of Technology (KTH), Stockholm, Sweden.

Schlatter, P., Brandt, L., de Lange, H. C. \& Henningson, D. S. 2008 On streak breakdown in bypass transition. Physics of Fluids 20 (10), 101505.

Schlatter, P. \& ÖRlü, R. 2010 Assessment of direct numerical simulation data of turbulent boundary layers. Journal of Fluid Mechanics 659, 116-126. 
Schlatter, P., Stolz, S. \& Kleiser, L. 2004 LES of transitional flows using the approximate deconvolution model. International Journal of Heat and Fluid Flow 25 (3), 549 - 558.

Schlatter, P., Stolz, S. \& Kleiser, L. 2006 Large-eddy simulation of spatial transition in plane channel flow. Journal of Turbulence 7, N33.

Schrauf, G. 2005 Status and perspectives of laminar flow. The Aeronautical Journal 109 (1102), 639-644.

Shelley, M. J. \& Zhang, J. 2011 Flapping and bending bodies interacting with fluid flows. Annual Review of Fluid Mechanics 43 (1), 449-465.

ShePherd, D. G. 1990 Historical development of the windmill. Tech. Rep.. NASA.

Spalart, P. R. 1988 Direct simulation of a turbulent boundary layer up to $\operatorname{Re}_{\theta}=1410$. Journal of Fluid Mechanics 187, 61-98.

Tchoufag, J., Fabre, D. \& Magnaudet, J. 2014a Global linear stability analysis of the wake and path of buoyancy-driven disks and thin cylinders. Journal of Fluid Mechanics $\mathbf{7 4 0}$, $278-311$

Tchoufag, J., Magnaudet, J. \& Fabre, D. $2014 b$ Linear instability of the path of a freely rising spheroidal bubble. Journal of Fluid Mechanics 751, R4.

Theodorsen, T. 1935 General theory of aerodynamic instability and the mechanism of flutter. Tech. Rep.. National Advisory Committee for Aeronautics; Langley Aeronautical Lab.; Langley Field, VA, United States.

Uzun, A. \& HussainI, M. Y. 2010 Simulations of vortex formation around a blunt wing tip. AIAA Journal 48, 1221-1234.

Visbal, M. R. 2011 Numerical investigation of deep dynamic stall of a plunging airfoil. AIAA Journal 49 (10), 2152 - 2170.

VisBaL, M. R. 2014 Analysis of the onset of dynamic stall using high-fidelity large-eddy simulations. In 52nd Aerospace Sciences Meeting, AIAA SciTech Forum. AIAA.

Visbal, M. R. \& Garmann, D. J. 2017 Numerical investigation of spanwise end effects on dynamic stall of a pitching NACA0012 wing. In 55th AIAA Aerospace Sciences Meeting, AIAA SciTech Forum. AIAA.

WiKIPEDIA, THE FREE ENCYCLOPEDIA 2017a North American P-51 Mustang. https://en.wikipedia.org/wiki/North_American_P-51_Mustang.

WIKIPEDIA, THE FREE ENCYCLOPEDIA $2017 b$ Wright Brothers. https://en.wikipedia.org/wiki/Wright_Flyer.

Williamson, C. \& Govardhan, R. 2004 Vortex-induced vibrations. Annual Review of Fluid Mechanics 36 (1), 413-455.

Xu, J. C., SEN, M. \& GAD-EL HAK, M. 1990 Low-Reynolds number flow over a rotatable cylindersplitter plate body. Physics of Fluids A: Fluid Dynamics 2 (11), 1925-1927.

Yuan, W., Poirel, D., Wang, B. \& Benaissa, A. 2015 Effect of freestream turbulence on airfoil limit-cycle oscillations at transitional Reynolds numbers. Journal of Aircraft 52 (4), 1214 1225 . 